\title{
Phonon effects in molecular transistors: Quantal and classical treatment
}

\author{
A. Mitra, I. Aleiner and A. J. Millis \\ Department of Physics, Columbia University \\ 538 West 120th St, NY, NY 10027 \\ (Dated: October 22, 2018)
}

\begin{abstract}
We present a comprehensive theoretical treatment of the effect of electron-phonon interactions on molecular transistors, including both quantal and classical limits. We study both equilibrated and out of equilibrium phonons. We present detailed results for conductance, noise and phonon distribution in two regimes. One involves temperatures large as compared to the rate of electronic transitions on and off the dot; in this limit our approach yields classical rate equations, which are solved numerically for a wide range of parameters. The other regime is that of low temperatures and weak electron-phonon coupling where a perturbative approximation in the Keldysh formulation can be applied. The interplay between the phonon-induced renormalization of the density of states on the quantum dot and the phonon-induced renormalization of the dot-lead coupling is found to be important. Whether or not the phonons are able to equilibrate in a time rapid compared to the transit time of an electron through the dot is found to affect the conductance. Observable signatures of phonon equilibration are presented. We also discuss the nature of the low- $\mathrm{T}$ to high- $\mathrm{T}$ crossover.
\end{abstract}

\section{INTRODUCTION}

In recent years it has become possible to fabricate devices in which the active element is a very small organic molecule ${ }^{1}$. Such a device may be thought of as a 'quantum dot': a structure weakly coupled to the macroscopic charge reservoirs ('leads') and small enough that the discrete nature of the energy levels on the dot is important. Quantum dots fabricated using conventional semiconductor technology have been extensively studied experimentally ${ }^{2}$ and theoretically ${ }^{3}$. However, the use of small molecules may lead to new physics. In particular, as electrons are added or removed from a small molecule, both the shape of the molecule and its position relative to the leads may be altered. The energies associated with these changes are not small, and the time scales may be comparable to those related to the flow of electrons into and out of the molecule. Interesting recent data indicate that these effects may lead to observable structures in the conductance spectra of the dot $\stackrel{4.5 .6}{ }$.

The shape change may be thought of as a coupling of electrons on the molecule to phonon modes of the molecule, while the position change corresponds to phonon dependent tunneling matrix elements. The subject of electron-phonon coupling in quantum dots has received much theoretical attention ${ }^{7.8 .9,10.11 .12 .13 .14 .15 .16}$. In an early important work Glazman and Shekhter obtained analytic expressions for the transmission probability through the dot under conditions that the phonons are always in equilibrium ${ }^{7}$. Their results for the transmission go a long way towards describing the behavior of the phonon-coupled system when one is far from resonance. However their treatment neglects the phonon renormalization of the dot-lead coupling, and thus gives rise to a zero bias conductance at resonance that is smaller than the value predicted by the Breit-Wigner formula (this situation was not the main interest of $\mathrm{f}^{\mathbf{7}}$ ). (These issues were also recently discussed by Flensberg ${ }^{15}$ ). The lack of renormalization of the dot-lead coupling appears in the treatment carried out by other authors as well ${ }^{18,9,10,14}$, and in addition some authors assert (incorrectly, we believe) that phonon sidebands may be observed even in the linear-response conductance $e^{9.10}$ by tuning the gate voltage.

In this paper we revisit the problem of the phonon coupled dot. We present a comprehensive formalism valid both in the classical and quantal limits which resolves the ambiguities in the present literature. We also use this formalism to address new issues related to the behavior of this system under strongly non-equilibrium conditions in the quantal and classical regime. We further study the nature of the quantal-classical crossover and the noise spectra. An important feature of our results in the quantum regime is that the conductance peak height at resonance is unchanged by electron-phonon interactions.

A recent paper by McCarthy et al 11 treats the problem of the phonon coupled dot in the high- $\mathrm{T}$ classical regime and for phonons that couple to the leads. They present results for the regime of "equilibrated" phonons strongly coupled to a heat bath. The high- $\mathrm{T}$ regime of our work is similar to that of reference ${ }^{11}$, but we also study the physics of out of equilibrium phonons.

Aji et al ${ }^{12}$ have studied the model under off-resonant conditions when the conductance is very low and the current is due to elastic/inelastic cotunneling. They study the phonon sidebands in the case of equilibrated and unequilibrated phonons, for the case of an electron coupled to a molecular vibrational mode (our model) and also the case of a phonon dependent tunneling amplitude. In our analysis, the exact treatment of the leads automatically takes into account cotunneling processes, while the perturbative approximation restricts our analysis to details of the first phonon sideband. We show how thermal effects wash out cotunneling.

The paper is organized as follows. In Section II we describe the model (sub-section A), the important model parameters (sub-section B) and develop a density matrix 
formalism (sub-section C) that allows one to obtain the probability distribution for various states on the dot under out of equilibrium conditions. In Section-III we apply the high temperature approximation in the density matrix formalism and derive the rate equations for the dot occupation probabilities in the sequential tunneling regime (sub-section $\mathrm{A}$ ). We use these rate equations to calculate average current (sub-section B) and the dc noise power (sub-section C) as functions of gate and sourcedrain voltage for two limiting cases: phonons equilibrated to the external world independent of the electron occupation (phonon equilibration fast compared to dwell time of electron on molecule), and phonons uncoupled from the external world and responding only to on-dot electrons (phonon equilibration slow compared to dwell time of electron on dot). We find that in the case of phonons uncoupled from a bath, under certain bias conditions the phonon distribution can deviate far from equilibrium.

Section-IV deals with the low temperature quantal regime of the phonon coupled dot where we use the Keldysh Green's function technique to calculate the dccurrent and phonon distribution function to leading order in the ratio of (electron-phonon coupling)/(tunneling rate to the leads) for the two cases of slow and fast phonon equilibration rate. Section IV is split into subsection A that re-introduces the problem, sub-section B that derives expressions for the exact eigenstates in the absence of electron-phonon coupling. These eigenstates form a convenient basis for carrying out the perturbative Keldysh calculation which is outlined in sub-section C. Sub-sections D and E present results for I-V for the two cases of slow and fast phonon equilibration rates.

Finally Section V studies the crossover from low- $\mathrm{T}$ to high-T regimes and section VI is a summary of our work and its relation to already existing literature.

\section{MODEL, PARAMETERS AND FORMALISM}

\section{A. Model}

We consider the case of a molecule with a single level of degeneracy $d_{g}$ coupled to two leads, which we label as 'left (L) ' and 'right (R)'. We suppose that the electrons are coupled to two different kinds of phonons; an internal vibrational mode of the molecule of frequency $\omega_{0}$, which couples to the local charge, and a phonon mode labeled by a displacement operator $\hat{z}$, that accounts for the oscillations of the dot in an external confining potential of parabolicity $K$. This phonon mode does not couple directly to the charge on the dot, but results in an explicit $\hat{z}$ dependence in the left and right tunneling matrix amplitudes $t_{i, k, \alpha}\{\hat{z}\}$. The full Hamiltonian is therefore,

$$
H=H_{D}+H_{\text {leads }}+H_{t}
$$

with

$$
\begin{aligned}
H_{D} & =\epsilon n_{d}+\frac{U}{2} n_{d}\left(n_{d}-1\right)+\lambda \omega_{0}\left(b^{\dagger}+b\right) n_{d} \\
& +\frac{p_{z}^{2}}{2 M}+\frac{1}{2} K z^{2}+\omega_{0} b^{\dagger} b \\
H_{\text {leads }} & =\sum_{k, \alpha=L, R} \epsilon_{k} a_{\alpha, k}^{\dagger} a_{\alpha, k} \\
H_{t} & =\sum_{\alpha=L, R, k, \sigma, i=1, d_{g}} t_{i, k, \alpha}\{\hat{z}\} a_{\alpha, k}^{\dagger} d_{i, \sigma}+h . c
\end{aligned}
$$

where $\sigma$ labels the spin index. Fig. [1]shows the schematic of the energies considered.

Here the number of electrons on the molecule, $n_{d}$, is given by

$$
n_{d}=\sum_{i=1, d_{g}, \sigma} d_{i, \sigma}^{\dagger} d_{i, \sigma},
$$

and the parameter $U$ is the charging energy of the molecule. We have defined the zero-phonon state of the vibrational mode of the molecule to be the ground state when $n_{d}=0$, and we neglected anharmonicity in the lattice part of the Hamiltonian (such anharmonicity is of course induced by the electron-phonon coupling and an intrinsic anharmonicity could easily be added).

The dot-lead coupling $t_{i, k, \alpha}$ means that $\left[H, n_{d}\right] \neq 0$; in the absence of electron-phonon and many-body physics this implies an inverse lifetime for decay of an electron in state $i$ on the dot into a state of energy $\epsilon$ in lead $\alpha$,

$$
\Gamma_{i, \alpha}(\epsilon)=2 \pi \sum_{k}\left|t_{i, k, \alpha}(\hat{z}=0)\right|^{2} \delta\left(\epsilon-\epsilon_{i, k}\right)
$$

The density matrix $\rho$ of the full Hamiltonian $H$ obeys the equation of motion

$$
\frac{d \rho(t)}{d t}=-i[H, \rho(t)]
$$

The current $I$ and the noise $S$ through the lead $\alpha$ are given by

$$
\begin{aligned}
\left\langle I_{\alpha}(t)\right\rangle & =\operatorname{Tr} \hat{\rho}(t) \hat{I}_{\alpha} \\
S_{\alpha}(t) & =\operatorname{Tr} \hat{\rho}(t)(\delta I(t) \delta I(0)+\delta I(0) \delta I(t))
\end{aligned}
$$

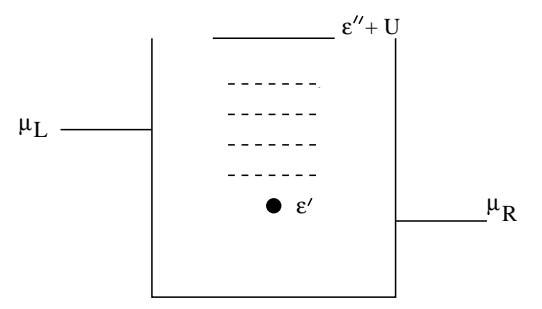

FIG. 1: Energy level diagram. $\mu_{L, R}$ represent the chemical potential in the left and right leads respectively, while $\epsilon^{\prime}$ represents the ground state of the singly occupied dot and the dashed lines indicate phonon excitations of the singly occupied dot. The solid line indicates the energy $\epsilon^{\prime \prime}+U$ of the doubly occupied dot. 
where $\delta I(t)=I(t)-\langle I\rangle$ and the current operator through the $\alpha$ lead is given by

$$
\begin{aligned}
\hat{I}_{\alpha} & =\frac{d N_{\alpha}}{d t}=i\left[H, N_{\alpha}\right] \\
& =i \sum_{k, \sigma, i}\left(t_{i, \alpha}(\hat{z}) a^{\dagger}{ }_{\alpha, k, \sigma} d_{i}-h . c\right)
\end{aligned}
$$

$N_{\alpha}=\sum_{k, \sigma} a_{k, \sigma}^{\dagger} a_{k, \sigma}$ being the number operator for the $\alpha$ lead.

\section{B. Parameters and regimes}

The behaviour of the models we consider is specified by two important dimensionless parameters: the ratio of the temperature $T$ to a typical decay time $\Gamma$, and the product of the dimensionaless coupling $\lambda$ and the ratio of the phonon frequency $\omega_{0}$ to $\Gamma$. For large values of $T / \Gamma$ a classical rate equation analysis may be employed for all values of $\lambda \omega_{0} / \Gamma$; this is the subject of Section III. For small values of $T / \Gamma$ a quantal treatment is required. Section IV reports results for low T, obtained using perturbative calculations valid for $\lambda \omega_{0} / \Gamma \leq 1$, while Section $\mathrm{V}$ treats the quantal-classical crossover, also in the $\lambda \omega_{0} / \Gamma \leq 1$ regime. The quantal strong coupling regime $\left(T / \Gamma<1, \lambda \omega_{0} / \Gamma>1\right)$ is a challenging problem left for future reserach. The different regimes and the sections treating them are shown in Fig. 2 .

\section{Formalism}

The essential assumption in the theory of molecular devices is that the leads are in equilibrium independent of the state of the molecule. In order to implement this assumption it is often convenient to define a projected density matrix $\rho_{s}$

$$
\rho_{s}=\operatorname{Tr}_{\text {leads }}\{\rho(t)\} \otimes \rho_{\text {leads }}
$$

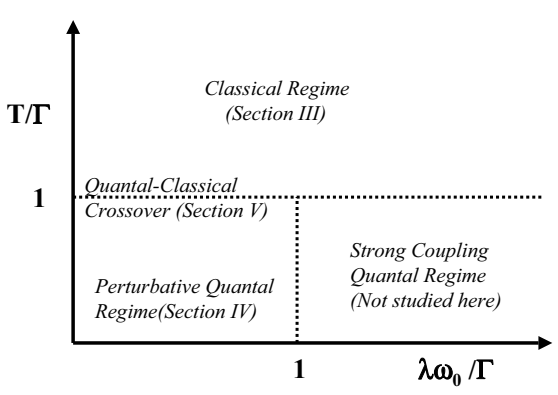

FIG. 2: Overview of different regimes studied. where $\rho_{\text {leads }}$ is the density matrix of the left and right leads that are in thermal equilibrium at some specified chemical potentials $\left(\mu_{L}\right.$ and $\left.\mu_{R}\right)$. The density matrix of the dot (also known as the reduced density matrix) is therefore given by

$$
\rho_{D}=T r_{\text {leads }} \rho_{s}
$$

and for our model corresponds to projecting the full density matrix to the smaller subspace of the degrees of freedom of the dot electrons and the two types of phonon modes.

A complementary density matrix $\rho_{t}$ may also be defined such that

$$
\rho_{t}=\rho-\rho_{s}
$$

from which it follows that

$$
\operatorname{Tr}_{\text {leads }} \rho_{t}=0
$$

It is desirable to obtain the reduced density matrix of the $\operatorname{dot} \rho_{D}$ because its diagonal components relate directly to the occupation probabilities of the various states of the dot, and various expectation values (such as the current and noise) may be expressed in terms of components of $\rho_{D}$.

In what follows we outline a general scheme for obtaining the reduced density matrix ${ }^{17}$. The equation of motion for the full density matrix, Eq. 7 implies

$$
\begin{aligned}
\frac{d \rho_{s I}(t)}{d t} & =\left(\operatorname{Tr}_{\text {leads }} \frac{d \rho_{I}(t)}{d t}\right) \otimes \rho_{\text {leads }} \\
& =-i\left(\operatorname{Tr}_{\text {leads }}\left[H_{t I}(t), \rho_{\text {It }}(t)\right]\right) \otimes \rho_{\text {leads }} \\
\frac{d \rho_{t I}(t)}{d t} & =\frac{d\left(\rho_{I}(t)-\rho_{s I}(t)\right)}{d t} \\
& =-i\left[H_{t I}(t), \rho_{s I}(t)+\rho_{t I}(t)\right] \\
& +i\left(\operatorname{Tr}_{\text {leads }}\left[H_{t I}, \rho_{t I}(t)\right]\right) \otimes \rho_{\text {leads }}
\end{aligned}
$$

We have used the interaction representation defined by $\hat{O}_{I}(t)=e^{i\left(H_{D}+H_{\text {leads }}\right) t} \hat{O}(t) e^{-i\left(H_{D}+H_{\text {leads }}\right) t}$.

In specifying the solution of Eqns. 15] and [16] we require an initial condition. There are two common choices:(i) at the initial time, $\hat{\rho}$ corresponds to an equilibrium ensemble for $H$ with $\mu_{L}=\mu_{R}$, (ii) at the initial time $\left(t_{i}\right)$ the dot and leads are decoupled $\left(H_{t}=0\right)$ so that $\rho\left(t_{i}\right)=\rho_{D} \otimes \rho_{\text {leads }}$ with $\rho_{D}$ and $\rho_{\text {leads }}$ the equilibrium density matrices corresponding to the uncoupled problem. We shall be interested in steady state, so we will take the initial time $t_{i}=-\infty$. Further we shall be interested in cases in which the leads are not strongly affected by the presence of the dot. In this case the boundary condition (ii) is most convenient, but we note that if orthogonality effects or Luttinger liquid renormalizations of tunneling amplitudes are important then this choice may be less convenient.

Choosing boundary condition (ii), Equation [16] being a linear equation in $\rho_{t I}(t)$ may be formally solved as

$$
\rho_{t I}(t)=-i \int_{-\infty}^{\infty} d t^{\prime} K_{I}\left(t, t^{\prime}\right)\left[H_{t I}\left(t^{\prime}\right), \rho_{s I}\left(t^{\prime}\right)\right]
$$


where $K_{I}\left(t, t^{\prime}\right)$ obeys the following operator equation,

$$
\begin{gathered}
\frac{d K_{I}\left(t, t^{\prime}\right) \bullet}{d t}+i\left[H_{t I}, K_{I}\left(t, t^{\prime}\right) \bullet\right] \\
-i\left(\operatorname{Tr}_{\text {leads }}\left[H_{t I}, K_{I}\left(t, t^{\prime}\right) \bullet\right) \otimes \rho_{\text {leads }}=\delta\left(t-t^{\prime}\right)\right.
\end{gathered}
$$

where the symbol $\bullet$ denotes the operators acted on by $K_{I}$. The combination of the second and third terms on the 1.h.s. of Eq. 18 correspond to processes in which the particle distribution in the leads differ from the equilibrium one. When substituted back in Eq. 17 these terms produce correlations between the dot and lead variables. Processes corresponding to these correlations have been discussed in detail by Scholler and co-workers ${ }^{24}$, however their importance for the present problem is unclear.

Substituting Eq. 17 into Eq. 15] leads to

$$
\frac{d \rho_{D I(t)}}{d t}=-\operatorname{Tr}_{\text {leads }} \int_{-\infty}^{\infty} d t^{\prime}\left[H_{t I}(t), K_{I}\left(t, t^{\prime}\right)\left[H_{t I}\left(t^{\prime}\right), \rho_{s I}\left(t^{\prime}\right)\right]\right]
$$

Eq. [19]is a generalized Master equation that under steady state conditions (setting the l.h.s. of Eq. 19 to zero) can be written in the following form,

$$
0=\sum_{j} P^{j} R_{j \rightarrow i}
$$

where $\left\langle i\left|\rho_{D}\right| i\right\rangle=P^{i}$, the probability of being in the ith dot state. (Under steady state conditions off-diagonal elements of the density matrix vanishes except in the case of accidental degeneracies). Conservation of probability requires $\sum_{i} R_{j \rightarrow i}=0$, from which it follows that

$$
0=\sum_{j \neq i} P^{j} R_{j \rightarrow i}-P^{i} \sum_{j \neq i} R_{i \rightarrow j}
$$

so that the quantities $R_{j \rightarrow i}$ may be interpreted as the various in-scattering and out-scattering rates.

A formal expression for the current may also be derived by using Eq. $\left[8\right.$ and observing that $\operatorname{Tr} \hat{I} \rho_{s}=0$, so that

$$
\begin{aligned}
\langle I\rangle & =\operatorname{Tr} \hat{I} \rho_{t} \\
& \left.=-i \operatorname{Tr} \int_{-\infty}^{\infty} d t^{\prime}\left[I_{I}(t) K_{I}\left(t, t^{\prime}\right)\right), H_{t I}\left(t^{\prime}\right)\right] \rho_{D I}\left(t^{\prime}\right) \otimes \rho_{\text {leads }}
\end{aligned}
$$

Eqns. 21 and 22 are our basic results. Further analysis depends on the specifics of the system and of the approximation chosen. At temperature $\mathrm{T}$, only times $t<1 / T$ are relevent, so at sufficiently high $T$, we may approximate $K_{I}$ by its short time behavior,

$$
K_{I}\left(t, t^{\prime}\right) \rightarrow \theta\left(t-t^{\prime}\right)
$$

Indeed making this substitution in Eq. 17 leads to the following expression for $\rho_{t}$ in the Heisenberg representation,

$$
\begin{gathered}
\rho_{t}=-i \int_{-\infty}^{t} d t^{\prime} e^{-i\left(H_{D}+H_{\text {leads }}\right)\left(t-t^{\prime}\right)}\left[H_{t}, \rho_{s}\left(t^{\prime}\right)\right] \\
e^{i\left(H_{D}+H_{\text {leads }}\right)\left(t-t^{\prime}\right)}
\end{gathered}
$$

The above expression for $\rho_{t}$ when substituted in the first line of Eq. $[22$ leads to the widely accepted MeirWingreen ${ }^{18}$ expression for the current,

$$
\begin{gathered}
I_{L}=\int_{-\infty}^{t} d t^{\prime} \int d \epsilon N(\epsilon) \operatorname{Im}\left\{e^{i \epsilon\left(t-t^{\prime}\right)} G^{<}\left(t, t^{\prime}\right)+f_{L}(\epsilon \nmid 25)\right. \\
\left.e^{i \epsilon\left(t-t^{\prime}\right)} G^{R}\left(t, t^{\prime}\right)\right\}
\end{gathered}
$$

where $N(\epsilon)$ is the density of states, and the Green's functions are defined as

$$
\begin{aligned}
& G^{R}\left(t, t^{\prime}\right)=-i \theta\left(t-t^{\prime}\right)\left\langle\left\{ d(t) \hat{t}_{k L}\{\hat{z}(t)\}, d^{\dagger}\left(t^{\prime}\right) \hat{t}_{k L}^{\dagger}\left\{\hat{z}\left(t^{\prime}\right)\right\}(26)\right.\right. \\
& G^{<}\left(t, t^{\prime}\right)=i\left\langle d^{\dagger}\left(t^{\prime}\right) \hat{t}_{k L}^{\dagger}\left\{\hat{z}\left(t^{\prime}\right)\right\} d(t) \hat{t}_{k L}\{\hat{z}(t)\}\right\rangle
\end{aligned}
$$

In general the second and third terms in Eq. $18 \mathrm{im}-$ ply $K_{I}(t)=\theta(t) k(t)$ where $k \neq 1$, suggesting the Meir-Wingreen formula is incomplete. However we suspect that in the present problem in which there is no backscattering and no "excitonic" interaction between dot and lead variables, correlations corresponding to $i\left[H_{t I},.\right]-i \operatorname{Tr}_{\text {leads }}\left[H_{t I},.\right] \otimes \rho_{\text {leads }} \neq 0$ (Eq. [18) are irrelevant: any such configuration simply propagates away from the lead and does not return. Some supporting evidence for this argument is provided by the exact diagonalization results of Section III; however the issue warrants further investigation.

For the simpler case of left and right phonon independent tunneling amplitudes the Green's functions in Eq.25 are simply the dot d-electron correlator. Wingreen et al ${ }^{18}$ showed that in this scenario and for the case of left and right tunneling amplitudes that are proportional to each other (but with non-trivial on-dot interactions), the current through a single resonant level simplifies to

$$
I=\int \frac{d \epsilon}{2 \pi} \frac{\Gamma_{L}(\epsilon) \Gamma_{R}(\epsilon)}{\Gamma_{L}(\epsilon)+\Gamma_{R}(\epsilon)} A(\epsilon)
$$

where $A(\epsilon)=i\left(G_{d}^{R}(\epsilon)-G_{d}^{A}(\epsilon)\right)$, with $G_{d}^{R}\left(t, t^{\prime}\right)=-i \theta(t-$ $\left.t^{\prime}\right)\left\langle\left\{d(t), d^{\dagger}\left(t^{\prime}\right)\right\}_{+}\right\rangle$. and $\Gamma_{L / R}(\epsilon)=2 \pi \sum_{k} t_{k, L / R}^{2} \delta\left(\epsilon_{k}-\epsilon\right)$.

When the temperature is the highest energy scale in the problem, approximation of Eq. 23 becomes exact, and further the Green's functions in Eq. 25] are replaced by their short time behaviour ${ }^{19}$. This shall be explicitly demonstrated in the next section where we will derive rate equations for the on-dot probability distribution within the sequential tunneling regime. Following this, in Section IV we shall carry out the Meir-Wingreen prescription for calculating the current for general temperatures.

\section{HIGH-TEMPERATURE APPROXIMATION: RATE EQUATIONS}

\section{A. Formalism}

In this section we shall carry out a high-T analysis for the simple case where the only phonon mode the electron degrees of freedom couple to is the on-dot vibrational 
mode. (The case of the phonons coupled to leads has been discussed is some detail by McCarthy et al ${ }^{11}$ within the high temperature approximation).

It is often convenient to choose a representation which is diagonal in the dot degrees of freedom. In the present model this is achieved via a standard ${ }^{20}$ canonical transformation. Defining $S=\lambda\left(\sum_{i, \sigma} d_{i, \sigma}^{\dagger} d_{i, \sigma}\right)\left(b^{\dagger}-b\right)$ and transforming all operators $O$ via $e^{S} O e^{-S}$ leads to a transformed Hamiltonian $H^{\prime}=H_{d o t}^{\prime}+H_{t}^{\prime}+H_{\text {leads }}$ with

$$
\begin{aligned}
& H_{D}^{\prime}=\varepsilon^{\prime} n_{d}+\omega_{0} \tilde{b}^{\dagger} \tilde{b}+\frac{\tilde{U}}{2} n_{d}\left(n_{d}-1\right) \\
& H_{t}^{\prime}=\sum_{a=L, R, i} t_{i, a} \sum_{p, \sigma}\left(\widehat{X} a_{a p \sigma}^{\dagger} d_{i, \sigma}+H . c .\right)
\end{aligned}
$$

where the transformed phonon operator $\tilde{b}=b-$ $\lambda \sum_{i, \sigma} d_{i, \sigma}^{\dagger} d_{i, \sigma}$, so that the phonon ground state depends on the dot occupancy. Moreover $\epsilon^{\prime}=\epsilon-\lambda^{2} \omega_{0}$ is the 'polaron shift' in the energy for adding one electron to the molecule and the interaction parameter $U$ is also renormalized, but as we shall focus here on $U \rightarrow \infty$ we do not write the renormalization explicitly. The crucial phonon renormalization of the electron-lead coupling is given by

$$
\widehat{X}=\exp \left[-\lambda\left(\tilde{b}^{\dagger}-\tilde{b}\right)\right]
$$

The high-T approximation proceeds from Eq. 24 by making the Markov approximation which involves replacing $\rho_{s}\left(t^{\prime}\right)$ in the integrand in Eq. 24] by $\rho_{s}(t)$. After substituting for $\rho_{t}$ in Eq. [15] it is also convenient to replace $\int_{-\infty}^{t}=\frac{1}{2} \int_{-\infty}^{\infty}$, which amounts to absorbing any level shifts induced by the dot-lead coupling into the bare values of the parameters. Following this we obtain

$$
\begin{gathered}
\frac{d \rho_{s}(t)}{d t}=-i\left[H_{D}+H_{\text {leads }}, \rho_{s}\right]-\frac{1}{2} \int_{-\infty}^{\infty} d t^{\prime} \\
{\left[H_{t}, e^{-i\left(H_{D}+H_{\text {leads }}\right)\left(t-t^{\prime}\right)}\left[H_{t}, \rho_{s}(t)\right] e^{i\left(H_{D}+H_{\text {leads }}\right)\left(t-t^{\prime}\right)}\right]}
\end{gathered}
$$

On the assumption that orthogonality effects may be neglected, we may formally take the trace over the lead degrees of freedom and in the process arrive at coupled equations of motion for the various occupation probabilities of the dot. We outline the calculation in detail for one of the 4 terms that one gets on opening up the commutator in Eq. 32 .

$$
\begin{gathered}
\operatorname{Tr}_{\text {leads }} \frac{\left(d \rho_{\text {leads }} \otimes \rho_{D}\right)}{d t} \\
=-\frac{1}{2} \int_{-\infty}^{\infty} d t^{\prime} \operatorname{Tr}_{\text {leads }} H_{t} e^{-i\left(H_{D}+H_{\text {leads }}\right)\left(t-t^{\prime}\right)} \\
H_{t} \rho_{\text {leads }} \otimes \rho_{D} e^{i\left(H_{D}+H_{\text {leads }}\right)\left(t-t^{\prime}\right)}+\ldots
\end{gathered}
$$

Using the following relations

$$
\begin{aligned}
\operatorname{Tr}_{\text {leads }} \rho_{\text {leads }} & =1 \\
\operatorname{Tr}_{\text {leads }}\left(\rho_{\text {leads }} a_{\alpha, k_{1}}^{\dagger} a_{\beta, k_{2}}\right) & =\delta_{\alpha, \beta} \delta_{k_{1}, k_{2}} f\left(\epsilon_{k}-\mu_{\alpha}\right) \\
\operatorname{Tr}_{\text {leads }}\left(\rho_{\text {leads }} a_{\alpha, k_{1}} a_{\beta, k_{2}}^{\dagger}\right) & =\delta_{\alpha, \beta} \delta_{k_{1}, k_{2}}\left(1-f\left(\epsilon_{k}-\mu_{\alpha}\right)\right)
\end{aligned}
$$

we obtain

$$
\begin{aligned}
\frac{d \rho_{D}}{d t} & =-\frac{1}{2} \int_{-\infty}^{\infty} d t^{\prime} \sum_{k, \alpha=L, R, i, j, \sigma} t_{\alpha}^{2} f_{k, \alpha} e^{i \epsilon_{k, \alpha}\left(t-t^{\prime}\right)} d_{i \sigma} \\
& X e^{-i H_{d}\left(t-t^{\prime}\right)} d_{j \sigma}^{\dagger} X^{\dagger} \rho_{D} e^{i H_{D}\left(t-t^{\prime}\right)} \\
& +t_{\alpha}^{2}\left(1-f_{k, \alpha}\right) e^{-i \epsilon_{k, \alpha}\left(t-t^{\prime}\right)} \\
& d_{i \sigma}^{\dagger} X^{\dagger} e^{-i H_{D}\left(t-t^{\prime}\right)} d_{j \sigma} X \rho^{D} e^{i H_{D}\left(t-t^{\prime}\right)}+\cdots
\end{aligned}
$$

We may now identify the probability $P_{m}^{n}$ of the dot being in a state with $\mathrm{n}$ electrons and $\mathrm{m}$ phonons as,

$$
P_{m}^{n}=\left\langle n, m\left|\rho_{D}\right| n, m\right\rangle
$$

Note that while $\rho_{D}$ is always diagonal in the dot electron number, it may be off-diagonal in the phonon number (due to the presence of the $X$ operators in Eq. 35). However such terms are negligibly small in comparision to the components of $\rho_{D}$ that are diagonal in phonon number, and are therefore neglected in our analysis. That is also the reason why we have dropped the first term in Eq. 32 in the next set of equations.

We are now in a position to write rate (Master) equations for the electron- phonon joint probabilities, which take the form

$$
\begin{gathered}
\dot{P}_{q}^{n}=\sum_{a, q^{\prime}} f_{a}\left(\left(q-q^{\prime}\right) \omega_{0}+U(n-1)\right) \Gamma_{q, q^{\prime}}^{a} P_{q^{\prime}}^{(n-1)}(37) \\
+\left(1-f_{a}\left(\left(q^{\prime}-q\right) \omega_{0}+U n\right)\right) \Gamma_{q, q^{\prime}}^{a} P_{q^{\prime}}^{(n+1)} \\
-\left(1-f_{a}\left(\left(q-q^{\prime}\right) \omega_{0}+U(n-1)\right)\right) \Gamma_{q^{\prime}, q}^{a} P_{q}^{n} \\
-f_{a}\left(\left(q^{\prime}-q\right) \omega_{0}+U n\right) \Gamma_{q^{\prime}, q}^{a} P_{q}^{n}
\end{gathered}
$$

Note that in our notation the upper index in $P_{q}^{n}$ always refers to the electron number and the lower index the phonon number, while $f_{a}(x)$ is short form for the Fermi function evaluated at $f\left(x+\epsilon^{\prime}-\mu_{a}\right), \mu_{a}$ being the chemical potential of lead $a$.

Thus within the high- $\mathrm{T}$ approximation the rate for going from an $n$ electron and $q$ phonon state on the dot to an $n-1$ electron $q^{\prime}$ phonon state is $R_{q \rightarrow q^{\prime}}^{n \rightarrow n-1}=$ $\sum_{a=L, R} f_{a}\left(\left(q-q^{\prime}\right) \omega_{0}+U(n-1)\right) \Gamma_{q, q^{\prime}}^{a}$, where $\Gamma_{q^{\prime}, q}^{a}$ represents the transition rate involving hopping an electron from the dot to lead $a$ and changing the phonon occupancy from $q$ (measured relative to the ground state of $H_{D}^{\prime}$ with occupancy $n$ ) to $q^{\prime}$ (measured relative to the ground state of $H_{D}^{\prime}$ with occupancy $\left.n-1\right)$ and is equal to the transition rate involving hopping an electron from the lead $a$ to the dot and changing the phonon occupancy from $q$ (measured relative to the ground state of $H_{D}^{\prime}$ with occupancy $n-1$ ) to $q^{\prime}$ (measured relative to the ground state of $H_{D}^{\prime}$ with occupancy $n$ ). More explicitly ${ }^{20}$

$$
\Gamma_{q^{\prime}, q}^{a}=\Gamma_{a}\left|<q^{\prime}\right| X|q>|^{2}
$$

The matrix element can be computed by standard methods ${ }^{20} ;$ its absolute value $|<q| X\left|q^{\prime}>\right|^{2} \equiv X_{q q^{\prime}}^{2}$ 
is symmetric under interchange of $q$ and $q^{\prime}$ and is

$$
X_{q<q^{\prime}}^{2}=\left|\sum_{k=0, q} \frac{\left(-\lambda^{2}\right)^{k}\left(q ! q^{\prime} !\right)^{1 / 2} \lambda^{\left|q-q^{\prime}\right|} e^{-\lambda^{2} / 2}}{(k) !\left((q-k) !\left(k+\left|q^{\prime}-q\right|\right) !\right.}\right|^{2}
$$

As interesting special cases, we write several lowest operators:

$$
\begin{array}{cc}
X_{0 n} & =e^{-\lambda^{2} / 2} \frac{\lambda^{n}}{\sqrt{n !}} \\
X_{11} & =\left(1-\lambda^{2}\right) e^{-\lambda^{2} / 2} \\
X_{21} & =\sqrt{2} \lambda\left(1-\frac{\lambda^{2}}{2}\right) e^{-\lambda^{2} / 2} \\
X_{22} & =\left(1-2 \lambda^{2}+\frac{\lambda^{4}}{2}\right) e^{-\lambda^{2} / 2}
\end{array}
$$

Observe that for certain values of $\lambda$ some of the matrix elements vanish. This unusual behavior is an interference phenomenon, which is slightly obscured by the notation. A state which has $q$ phonons excited above the ground state of the system with $n=0$ electrons is a superposition (with varying sign) of many multiphonon states, when viewed in the basis which diagonalizes the $n=1$ electron problem, and therefore the transition described by $X_{q q^{\prime}}$ is really a superposition of many different transitions, which for some values of $\lambda$ may destructively interfere. In several recent papers ${ }^{14}$ the phonon renormalization of the molecule-lead coupling is apparently omitted, or treated in an average manner which neglects the $q, q^{\prime}$ dependent structure.

\section{B. I-V characteristics}

In this subsection we shall discuss the I-V characteristics obtained from the solution of the high temperature rate equations for two extreme cases. One is a scenario where the phonons are not coupled to a bath and their number changes only when electrons hop on and off the dot. The second case is when the phonons are strongly coupled to a bath, and are always forced to be in equilibrium.

From Eq. 22] we obtain

$$
\langle I\rangle=\operatorname{Tr} \rho_{t}(t) I_{L}
$$

with

$$
\hat{I}_{\alpha=L / R}=i t_{L} \sum_{k}\left(a_{\alpha, k}^{\dagger} d X-d^{\dagger} X^{\dagger} a_{\alpha, k}\right)
$$

Using Eq. 24 for $\rho_{t}$ and following the same procedure of tracing out the metal degrees of freedom, we arrive at the following expression for the current through the lead $a$ in terms of the joint probability distribution functions,

$$
\begin{aligned}
I_{a}= & \sum_{n, q, q^{\prime}}\left(2 d_{g}-n\right) P_{q}^{n} f_{a}\left(\left(q^{\prime}-q\right) \omega_{0}+U n\right) \Gamma_{q, q}^{a}(46) \\
& -(n+1) P_{q}^{n+1}\left(1-f_{a}\left(\left(q-q^{\prime}\right) \omega_{0}+U n\right)\right) \Gamma_{q^{\prime}, q}^{a}
\end{aligned}
$$

where the sum on $n$ is from 0 to $\left(2 d_{g}-1\right), 2 d_{g}$ being the maximum occupation of the dot.

As an aside consider the simple case of spinless electron with no coupling to phonons with a non-degenerate dot level of energy $\epsilon_{d}$. In this case the rate equations give us the following probability for a singly occupied level,

$$
P^{1}=\frac{\Gamma_{L} f\left(\epsilon_{d}-\mu_{L}\right)+\Gamma_{R} f\left(\epsilon_{d}-\mu_{R}\right)}{\Gamma_{L}+\Gamma_{R}}
$$

while the current from Eq. 46] is simply

$$
I_{L}=\frac{\Gamma_{L} \Gamma_{R}}{\Gamma_{L}+\Gamma_{R}}\left\{f\left(\epsilon_{d}-\mu_{L}\right)-f\left(\epsilon_{d}-\mu_{R}\right)\right\}
$$

On comparing the above expression with the exact solution for the current obtained by Wingreen et al. (Eq. 28) we can explicitly see that the high-T approximation corresponds to assuming the spectral function is a deltafunction.

We shall now discuss the opposite limit, of phonons equilibrated to an independent heat bath, assumed to be at the same temperature as the leads. To implement this we force the probability distributions on the right hand side of Eq. 37 to have the phonon-equilibrium form $P_{q}^{n}=P^{n} e^{-q \omega_{0} / T}\left(1-e^{-\omega_{0} / T}\right)$. In the $U \rightarrow \infty$ limit this ansatz implies that the probability $P^{0}$ that the dot is empty is given by,

$$
P^{0}=\frac{\sum_{a, q, q^{\prime}} \Gamma_{q, q^{\prime}}^{a} e^{-q \omega_{0} / T} \bar{f}_{a, q, q^{\prime}}}{\sum_{a, q, q^{\prime}} 2 \Gamma_{q, q^{\prime}}^{a} e^{-q^{\prime} \omega_{0} / T} f_{a, q, q^{\prime}}+\Gamma_{q, q^{\prime}}^{a} e^{-q \omega_{0} / T} \bar{f}_{a, q, q^{\prime}}}
$$

where $\bar{f}_{a, q, q^{\prime}}=1-f_{a}\left(\left(q-q^{\prime}\right) \omega_{0}\right), f_{a, q, q^{\prime}}=1-\bar{f}_{a, q, q^{\prime}}^{(49)}$ while, $P^{1}=1-P^{0}$.

In general for both equilibrated and unequilibrated cases the rate equations may be written in the matrix form

$$
\dot{P}=\hat{M} P
$$

Therefore under steady state conditions $\left(\dot{P}_{n}=0\right)$, the problem reduces to finding the eigenvector corresponding to the zero eigenvalue of the matrix $\hat{M}$. We do this numerically. From these solutions we have computed the current. Representative results are shown in Fig. 3 which plots the low-T current as a function of $V_{s d}$ for two gate voltages: $V_{g}=0\left(\mu_{L}=-\mu_{R}\right.$, upper panel $)$ and $V_{g}=\frac{V_{s d}}{2}\left(\mu_{R}=0\right.$, lower panel $)$, for both equilibrated and unequilibrated phonons. (Note that the calculation is for large values of $U$ which correspond to negligible double occupation probabilty for the dot electrons).

Steps (broadened by $\mathrm{T}$ ) in the current associated with "phonon side-bands" are observed when the source-drain voltage passes through an integer multiple of the phonon frequency. However, in the opposite 'linear response' limit $V_{s d} \rightarrow 0$ (not shown), as $V_{g}$ is varied we find just one main step in the $I-V$-curve, as $V_{g}$ passes through 0 , and only very tiny structures (vanishing as 


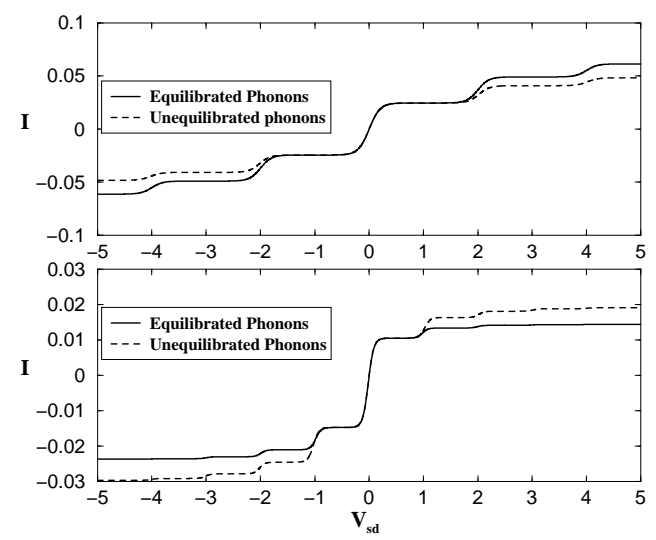

FIG. 3: Current $(I)$ vs source-drain voltage $V_{s d}$ for coupling constant $\lambda=1.0 \omega_{0}=1$ and $T=0.05$. Upper panel is for $V_{g}=0.0$, while lower panel is for $V_{g}=V_{s d} / 2, \mu_{R}=0 . I$ is in units of $\frac{e T}{\hbar}$

$e^{-\omega_{0} / T}$, which is the probability of the dot being empty with one phonon excited) when $V_{g}$ is a non-zero multiple of the phonon frequency. This result appears to differ from that stated by other authors $\$ .10$ who find phonon side bands as $V_{g}$ is swept at $V_{s d} \rightarrow 0$. The authors of $\underline{9,10}$ apparently neglected the fact that

the phonon side-bands "float" i.e., shift with the fermi level as $V_{g}$ is changed.

Fig. 3] reveals on first sight an apparently surprising result: for symmetric bias $\left(V_{g}=0\right)$ and for the coupling considered, the current is larger for equilibrated phonons than for the unequilibrated case, whereas for the strongly asymmetric case $\left(\mu_{R}=0\right)$, the opposite is true. This is surprising because one expects that in the unequilibrated case the phonons arrange themselves so as to maximize the current. To gain more insight into this phenomenon we have calculated the dependence of the ratio of currents for unequilibrated and equilibrated phonons on the coupling $\lambda$ for different degrees of bias asymmetry. We find that except for $\mu_{R}=0.0$ (the most asymmetric case) a minimum in the ratio occurs for a $\lambda \sim 1$. This behaviour may be traced back to Eq. 4143 which reveal that higher order "diagonal" ( $n$ phonon- $n$ phonon) matrix elements vanish for a $\lambda \sim 1$.

The steps in current may be conveniently parameterized by the height (or the area, as the width is simply proportional to $T$ ) of the corresponding peaks $G_{\max }$ in the differential conductance $G=d I / d V$. Ratios of peak heights (or areas) provide a convenient experimental measure of whether the phonons are in equilibrium. At low $\mathrm{T}$, the equilibrium phonon distribution corresponds to occupancy only of the $n=0$ phonon state, so the $\mathrm{n}$-th side band involves a transition from the 0 phonon to the $\mathrm{n}$ phonon state. Therefore the ratios of the peak heights or areas are controlled by ratios of $\left|X_{n 0}\right|^{2}$. In particular

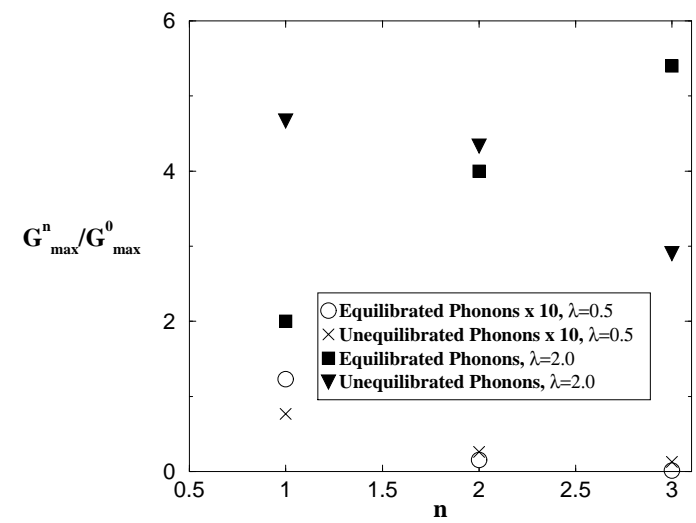

FIG. 4: Ratio of differential conductance $(G)$ peak heights for equilibrated and unequilibrated phonons and two different coupling strengths and $\mu_{L}=-\mu_{R}$. The points for $\lambda=0.5$ (open symbols) have been multiplied by 10 .

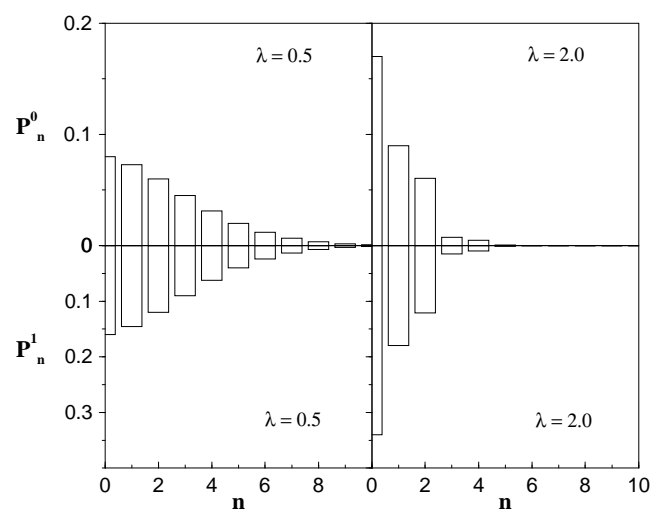

FIG. 5: Phonon probability distributions for two different electron-phonon coupling constants calculated for $\mu_{L}=$ $-\mu_{R}=2 \omega_{0}$.

Eqns. 40, 46] imply that if $\mu_{L}=-\mu_{R}$ and $T \ll \omega_{0}$,

$$
\left.\frac{G_{\max }^{n}}{G_{\text {max }}^{0}}\right|_{\text {equil }}=\frac{\left|X_{n 0}\right|^{2}}{2\left|X_{00}\right|^{2}}=\frac{\lambda^{2 n}}{2(n !)}
$$

Note that if $\mu_{L} \neq \mu_{R}$, then $\mu$ dependent changes in the occupation probabilities lead to additional, and not simply characterized $n$ dependence.

Deviations from this pattern imply non-equilibrium phonons. As illustration we display in Fig. 目 $G_{\max }$ values (normalized to the zero frequency peak) for equilibrium and non-equilibrium phonons and a weak and strong electron phonon coupling. One sees that in the non-equilibrium case the peak heights display a nonsystematic dependence on electron-phonon coupling and peak index, but that in general measurements of the $n=1$ and $n=2$ peaks reveal the effect clearly.

It is also of interest to consider how far out of equilibrium the phonon distribution may be driven. Fig. 5 shows the phonon occupation probabilities for weak and 


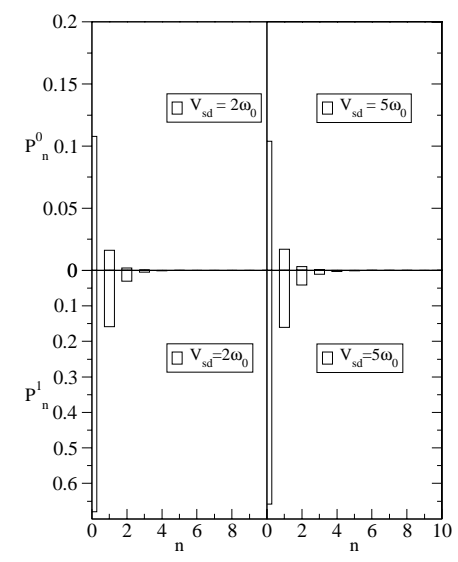

FIG. 6: Phonon probability distributions for weak electronphonon coupling $(\lambda=0.5)$ calculated for $\mu_{L}=2 \omega_{0}, \mu_{R}=0.0$ (left panel) and $\mu_{L}=5 \omega_{0}, \mu_{R}=0.0$ (right panel). Note the saturation in the probability distribution function which is also much closer to equilibrium. This situation is quite different for the same coupling constant and symmetric bias (see Fig. 5).

strong electron-phonon coupling and $V_{g}=0$. One sees immediately that the phonon distribution function is farther from equilibrium for weak couplings than for strong couplings. We associate this effect to the strong $\lambda$ dependence of operators $X_{0 n}$, (Eq. 40) which allows the system at large $\lambda$ to "jump down" from a highly excited state to one of low phonon occupancy. The deviation from equilibrium is largest for $V_{g}=0$ for similar reasons. Fig. [6] illustrates the scenario of non-zero gate voltage or asymmetric bias conditions $\mu_{L}=V_{s d}, \mu_{R}=0.0$. Here the phonon distribution for weak coupling saturates with bias to a value which is closer to its equilibrium distribution. As we shall show in Section IV, this gate voltage dependence of the non-equilibrium phonon distribution function is recovered in the quantal regime $\Gamma \gg T$ as well. Fig. 7 is the average phonon number $N_{p h}=\sum_{n, m} n P_{n}^{m}$ for moderate electron-phonon coupling. The steps in $N_{p h}-V_{s d}$ observed here coincide with the steps in I$\mathrm{V}$ and correspond to sequential (direct) tunneling. This is to be contrasted with the quantal regime (Section IV, Fig. 15] where $N_{p h}$ increases continously with bias due to higher order cotunneling processes.

\section{DC Noise characteristics}

Another important spectroscopic tool that is sensitive to the details of the electron-phonon coupling and to the phonons distribution is the current noise. In this subsection we outline the calculation of the dc current noise within the high-T approximation. Quite generally, the current noise $S_{L L}(t)$ through the left lead is given by the

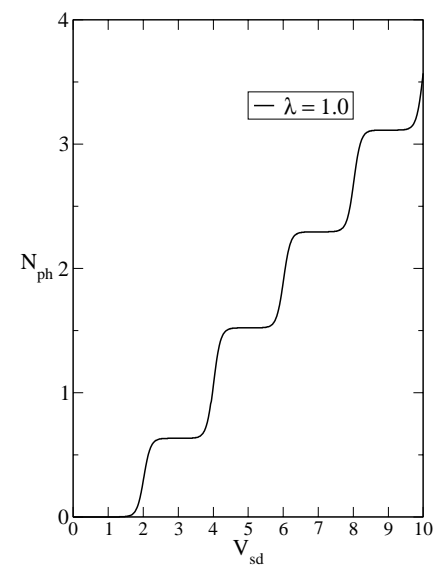

FIG. 7: Average phonon number under symmetric bias conditions, $\mu_{L}=-\mu_{R}$, electron-phonon coupling $\lambda=1.0$ and $\omega_{0}=20 T$.

following correlation function 17.21

$$
S_{L L}(t)=\frac{1}{2} \operatorname{Tr} \rho\left\{I_{L}(t) I_{L}(0)+I_{L}(0) I_{L}(t)\right\}-\left(\operatorname{Tr} \rho I_{L}\right)^{2}
$$

Using the fact that $I_{L}(t)=e^{i H t} I_{L}(0) e^{-i H t}$, the above expression for noise may be rewritten as

$$
S_{L L}(t)=\frac{1}{2} \operatorname{Tr} \lambda(t) I_{L}(0)
$$

where

$$
\lambda(t)=e^{-i H t}\left\{\left(I_{L}-\left\langle I_{L}\right\rangle\right) \rho+\rho\left(I_{L}-\left\langle I_{L}\right\rangle\right)\right\} e^{i H t}
$$

Since we are calculating the correlation of the same physical quantity at two different times, we expect $S(t)=$ $S(-t)$. Therefore we shall explicitly calculate $S(t)$ for positive times, for which we need to calculate the causal function $\lambda(t)$ that obeys the equation of motion

$$
\begin{aligned}
\frac{d \lambda(t)}{d t} & =-i[H, \lambda(t)]+\delta(t)\left\{\delta I_{L} \rho+\rho \delta I_{L}\right\}, t \geq 0(55) \\
& =0, \quad t<0
\end{aligned}
$$

where $\delta I_{L}=I_{L}-\left\langle I_{L}\right\rangle$. Note that $\operatorname{Tr} \lambda(0)=0$, and we expect $\lambda(t)$ to be traceless at all times. Also from charge conservation it follows that the noise across the left and right leads are equal $\left(S_{L L}(t)=S_{R R}(t)\right)$.

We now solve the equation of motion for $\lambda$ by decomposing $\lambda=\lambda_{s}+\lambda_{t}$, where $\lambda_{s}=\lambda_{D} \otimes \lambda_{\text {leads }}$ is diagonal in the dot and lead variables, while $\lambda_{t}$ is off-diagonal. After doing a similar decomposition for the density matrices $\rho=\rho_{s}+\rho_{t}$, the equation of motion for $\lambda$ (Eq. [55) may be re-written in a manner similar to that done for the 
equations of motion for $\rho$ in the previous section

$$
\begin{aligned}
\frac{d \lambda_{s}}{d t} & =-i\left[H_{t}, \lambda_{t}\right]+\delta(t)\left(I \rho_{t}+\rho_{t} I-2\langle I\rangle \rho_{s}\right) \\
\frac{d \lambda_{t}}{d t} & =-i\left[H_{D}+H_{\text {leads }}, \lambda_{t}\right]-i\left[H_{t}, \lambda_{s}\right] \\
& +\delta(t)\left(I \rho_{s}+\rho_{s} I-2\langle I\rangle \rho_{t}\right)
\end{aligned}
$$

Note that we have dropped the $\left[H_{D}+H_{m}, \lambda_{s}\right]$ term for the same reasons as before, namely that the matrix elements of this term is off-diagonal in the phonon number and is very small in comparision to the components of $\lambda_{s}$ that are diagonal in phonon number.

The solution for $\lambda_{t}$ from Eq. 57 is given by,

$$
\begin{aligned}
\lambda_{t}(t) & =-i \int_{-\infty}^{t} d t^{\prime} e^{-i\left(H_{D}+H_{\text {leads }}\right)\left(t-t^{\prime}\right)}\left[H_{t}, \lambda_{s}\left(t^{\prime}\right)\right] \quad(58) \\
& e^{i\left(H_{D}+H_{\text {leads }}\right)\left(t-t^{\prime}\right)}+\theta(t) e^{-i\left(H_{D}+H_{\text {leads }}\right) t} \\
& \left(I(0) \rho_{s}(0)+\rho_{s}(0) I(0)-2\langle I\rangle \rho_{t}(0)\right) e^{i\left(H_{D}+H_{\text {leads }}\right) t}
\end{aligned}
$$

Substituting Eq. 58 in Eq. .56 and going through the usual steps of extending the upper range of the integral to infinity, and making the Markov approximation (which involves pulling $\lambda$ out of the time integral), we arrive at the following matrix expression for the equation of motion for $\lambda$,

$$
\begin{aligned}
\frac{d \lambda_{D}}{d t} & =\hat{M} \lambda_{D}+\delta(t) h, t \geq 0 \\
& =0, t<0
\end{aligned}
$$

The matrix $\hat{M}$ is the same that enters in the equation of motion for $\rho_{D}$. In arriving at the expression for the vector $h$, we have approximated oscillating factors such as $e^{i \epsilon t} \sim 2 \pi \delta(t) \delta(\epsilon)$. Following this the vector $h$ has the structure

$$
\begin{gathered}
h_{q}^{n}=-2\left\langle I_{L}\right\rangle P_{q}^{n}+2 \sum_{q^{\prime}, n}\left(2 d_{g}-n\right) P_{q^{\prime}}^{n} \\
R_{q^{\prime}, q}^{L, n, n+1}-(n+1) P_{q^{\prime}}^{n+1} R_{q^{\prime}, q}^{L, n+1, n}
\end{gathered}
$$

where as before the sum on $n$ is from 0 to $2 d_{g}-1, d_{g}$ being the number of degenerate levels not counting spin. Note that we are using the following short-hand notation,

$$
\begin{array}{r}
R_{q, q^{\prime}}^{a, n+n+1}=f_{a}\left(\left(q^{\prime}-q\right) \omega_{0}+U n\right) \Gamma_{q, q^{\prime}}^{a} \\
R_{q^{\prime}, q}^{a, n+1, n}=\left(1-f_{a}\left(\left(q^{\prime}-q\right) \omega_{0}+U n\right)\right) \Gamma_{q, q^{\prime}}^{a}
\end{array}
$$

Moreover by using Eq. 46] it is easy to check that $h$ (whose components are given in Eq. 60) is traceless.

Now we shall rewrite the Eq. 53 explicitly in terms of the components of $\lambda_{D}$ and the steady state probabilities $P_{n}^{0}$ and $P_{n}^{1}$ (components of reduced density matrix $\rho_{D}$ ). After some algebra one finds that

$$
\begin{aligned}
S_{L L}(t) & =\frac{1}{2} \operatorname{Tr}_{m, D} I_{L} \lambda_{t}, t \geq 0 \\
& =-\left\langle I_{L}\right\rangle^{2}+\frac{1}{2}\left(S_{1 L}(t)+S_{2 L}(t)\right)
\end{aligned}
$$

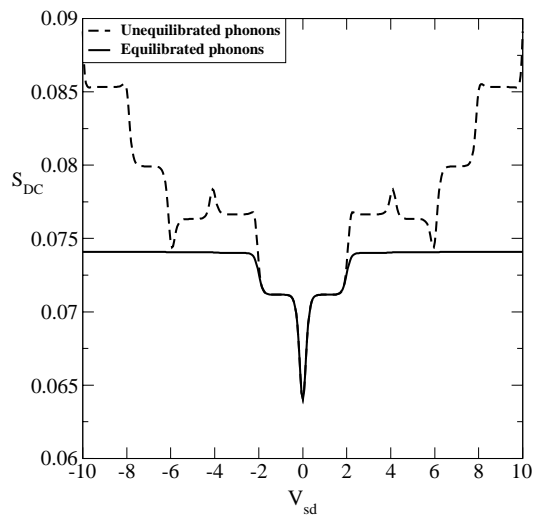

FIG. 8: DC Noise for $\lambda=0.2$ and under symmetric bias conditions $\mu_{L}=-\mu_{R}=V_{s d} / 2 . V_{s d}$ is in units of $\omega_{0}=20 T$. $S_{d c}$ is in units of $\frac{e^{2} T}{\hbar}$.

where $S_{L L}(-t)=S_{L L}(t)$, and

$$
\begin{gathered}
S_{1 L}(t)=\sum_{q, q^{\prime}, n}\left(2 d_{g}-n\right) \lambda_{q}^{n}(t) R_{q, q^{\prime}}^{L, n+1} \\
-(n+1) \lambda_{q}^{n+1}(t) R_{q, q^{\prime}}^{L, n+1, n}
\end{gathered}
$$

and

$$
\begin{gathered}
S_{2 L}(t)=2 \delta(t) \sum_{q, q^{\prime}, n}\left(2 d_{g}-n\right) P_{q}^{n}(t) R_{q, q^{\prime}}^{L, n+1} \\
+(n+1) P_{q}^{n+1}(t) R_{q, q^{\prime}}^{L, n+1, n}
\end{gathered}
$$

Note our notation $\lambda_{q}^{n}=\left\langle n, q\left|\lambda^{D}\right| n, q\right\rangle$ etc. where $\lambda^{D}=$ $\operatorname{Tr}_{\text {leads }} \lambda_{s}$. Also note that the $\delta(t)$ in the expression for $S_{2 L}(t)$ again arises from replacing oscillating factors $e^{i \epsilon t} \sim 2 \pi \delta(t) \delta(\epsilon)$.

We find it convenient to perform the following shift of variables $\lambda^{D} \rightarrow \lambda^{D}+2\left\langle I_{L}\right\rangle P$. This shift of variables does not affect the equations of motion Eq. 59 because $\hat{M} P=0$ in steady state. However this shift of variables cancels the $\left\langle I_{L}\right\rangle^{2}$ term in Eq. 63 .

Collecting all the terms we arrive at the following expression for the DC noise power

$$
\begin{gathered}
\tilde{S}_{d c}=2 \int_{-\infty}^{\infty} d t S_{L L}(t) \\
\tilde{S}_{d c}=2 \sum_{q, q^{\prime}, n}\left(2 d_{g}-n\right)\left(P_{q}^{n}+\tilde{\lambda}_{q}^{n}(0)\right) R_{q, q^{\prime}}^{L, n, n+1} \\
-(n+1)\left(-P_{q}^{n+1}+\tilde{\lambda}_{q}^{n+1}(0)\right) R_{q, q^{\prime}}^{L, n+1, n}
\end{gathered}
$$

where the components of $\tilde{\lambda}(\omega=0)$ are obtained from solving the matrix equation

$$
\tilde{\lambda}^{D}(\omega=0)=-M^{-1} h
$$


Note that the matrix $\hat{M}$ has the property that $\sum_{i} M_{i j}=$ 0 , so that one of its eigenvalues is 0 . However $h$ being traceless, $M^{-1} h$ is well defined.

Let us now look at the simple case of no electronphonon coupling and spinless electrons. In that case the matrix $\hat{M}$ acqires the simple $2 \times 2$ form (note that $f_{a}=1 /\left(1+e^{\left(\epsilon^{\prime}-\mu_{a}\right) / T}\right)$

$M=\left(\begin{array}{cc}-\left(\Gamma_{L} f_{L}+\Gamma_{R} f_{R}\right) & \Gamma_{L}\left(1-f_{L}\right)+\Gamma_{R}\left(1-f_{R}\right) \\ \Gamma_{L} f_{L}+\Gamma_{R} f_{R} & -\left(\Gamma_{L}\left(1-f_{L}\right)+\Gamma_{R}\left(1-f_{R}\right)\right)\end{array}\right)$

while the components of $\tilde{\lambda}(\omega=0)$ are given by

$$
\tilde{\lambda}^{0}=-\tilde{\lambda}^{1}=-\frac{h^{1}}{\Gamma_{L}+\Gamma_{R}}
$$

where

$$
h^{1}=2 \Gamma_{L} P^{0} f_{L}-2\left\langle I_{L}\right\rangle P^{1}
$$

The full expression for the dc-noise is

$$
\begin{aligned}
\tilde{S}_{d c}=2 \Gamma_{L}\left(P^{0} f_{L}\right. & \left.+P^{1}\left(1-f_{L}\right)\right)-4 \frac{\Gamma_{L}^{2}}{\Gamma_{L}+\Gamma_{R}} P^{0} f_{L} \\
& +4 \frac{\Gamma_{L}}{\Gamma_{L}+\Gamma_{R}}\left\langle I_{L}\right\rangle P^{1}
\end{aligned}
$$

We may now derive expressions for the dc noise in two limits. The first one is in the linear response regime $\mu_{L}=$ $\mu_{R}$ so that $P^{0}=1-f_{L}$ and $\left\langle I_{L}\right\rangle=0$. In that case,

$$
\tilde{S}_{d c}=4 \frac{\Gamma_{L} \Gamma_{R}}{\Gamma_{L}+\Gamma_{R}} f_{L}\left(1-f_{L}\right)
$$

which is the result expected from the fluctuation dissipation theorem $S_{d c}=4 T G$.

The other limit is $\mu_{L}=-\mu_{R}=e V / 2 \gg T$ so that by using the expression $P^{1}=\frac{\Gamma_{L} f_{L}+\Gamma_{R} f_{R}}{\Gamma_{L}+\Gamma_{R}}$ and setting to zero combinations such as $f_{L}\left(1-f_{L}\right)$ we obtain

$$
\tilde{S}(\omega=0)=2 \frac{\Gamma_{L}^{2}+\Gamma_{R}^{2}}{\left(\Gamma_{L}+\Gamma_{R}\right)^{2}}\left\langle I_{L}\right\rangle
$$

where $\left\langle I_{L}\right\rangle=\frac{\Gamma_{L} \Gamma_{R}}{\Gamma_{L}+\Gamma_{R}}$. The above expression gives the standard shot noise result when $\Gamma_{L} \ll \Gamma_{R}{ }^{21}$

The results for the noise for the case of phonons in equilibrium and the opposite case of phonons not coupled to any heat bath are illustrated in figures 8 (weak coupling) and 9 (strong coupling). The difference between the equilibrated and unequilibrated cases is more dramatic for smaller $\lambda$. For $\lambda=0.2$ while only one phonon side band is seen for the equilibrated case, very sharp phonon side bands are seen for the entire bias range when the phonon distribution is far out of equilibrium, with certain side bands appearing as peaks (rather than steps) associated with the suppression of noise.

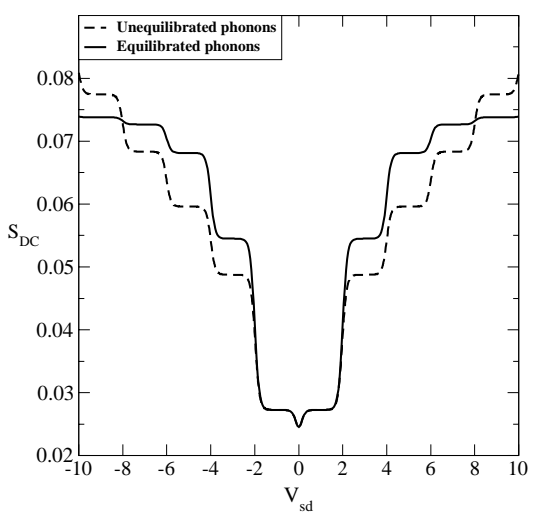

FIG. 9: DC Noise for $\lambda=1.0$ and under symmetric bias conditions $\mu_{L}=-\mu_{R}=V_{s d} / 2 . V_{s d}$ is in units of $\omega_{0}=20 T$. $S_{d c}$ is in units of $\frac{e^{2} T}{\hbar}$.

\section{QUANTUM THEORY OF TRANSPORT THROUGH A PHONON-COUPLED DOT}

\section{A. Overview}

In this section we present a fully quantum mechanical treatment of the simple limit of the model considered in previous sections. In order to carry out the calculation we restrict attention to a single non-degenerate level with no onsite coulomb $U$, and to low orders in perturbation theory in the electron-phonon coupling. The results shed light on the relation between the Green's function formalism natural in the quantal treatment, and the density matrix formalism natural in classical problems, and elucidate the quantal to classical crossover. For the reader's convenience we reproduce here the limit of Eqns. 233 and 4 which we study.

$$
\begin{aligned}
H_{e l} & =\epsilon_{0} d^{\dagger} d+\sum_{k, a=L, R} \epsilon_{k} a_{k, a}^{\dagger} a_{k, a} \\
& +\sum_{k, a=L, R} t_{k, a}\left(d^{\dagger} a_{k, a}+h . c\right) \\
H_{p h} & =\omega_{0} b^{\dagger} b \\
H_{e l-p h} & =\lambda \omega_{0}\left(b+b^{\dagger}\right) d^{\dagger} d
\end{aligned}
$$

Note that we have neglected the spin index, which may be restored in the final expressions for the current by simply multiplying by a factor of 2 .

We use two methods to analyze the above Hamilto- 
nian. One is the Keldysh Green's function method and the other is an explicit construction of the eigenstates and thus the density matrix. We note that the Green's function method directly computes the expectation value of operators at different times, bypassing the explicit construction of the density matrix. However equal time multiparticle Green's functions correspond to moments of the density matrix, and permit in principle its reconstruction.

The rest of the section is divided as follows. In subsection $\mathrm{B}$ we provide the exact solution in the absence of coupling to phonons. The eigenstates of this system will form the basis for the perturbative calculations that follow. In subsection $\mathrm{C}$ we outline the Keldysh calculation while in subsection $\mathrm{D}$ we present results for the special case when the phonon distribution is always in its ground state. In section $\mathrm{E}$ and $\mathrm{F}$ we generalize to the case when the phonons are allowed to deviate from equilibrium and also supplement our results obtained from the Keldysh Green's function technique by a perturbative calculation for the phonon density matrix that allows us to obtain the out of equilibrium phonon distribution function.

\section{B. Non-interacting dot and exact expressions for the current for an interacting dot}

Following standard methods ${ }^{20}$, the exact eigenstates of $H_{e l}$ (Eq. [75) can be easily obtained. The Hamiltonian after diagonalization is

$$
H_{e l}=\sum_{k, a=L, R} \epsilon_{k} \alpha_{k, a}^{\dagger} \alpha_{k, a}
$$

Note that while $a_{k, L / R}$ in Eq. 75 refer to states that live only on the left/right lead, an exact eigenstate of $H_{e l}$ has non-vanishing amplitude in both leads. We write the exact solution in a scattering state basis in which $\alpha_{k, a=L / R}$ refers to a running wave incident from the left/right lead with a certain amplitude of getting reflected back to the starting lead and a corresponding amplitude for transmission to the other side. The label $\mathrm{L} / \mathrm{R}$ now refers to the lead from which the particle is incident, and therefore determines the distribution function describing occupancy of the states. The dot and metal electron creation/annihilation operators are related to these exact (scattering) eigenstates as follows

$$
\begin{aligned}
a_{k, a} & =\sum_{k^{\prime}, b=L, R} \eta_{k a, k^{\prime} b} \alpha_{k^{\prime}, b} \\
d & =\sum_{k, a=L, R} \nu_{k, a} \alpha_{k, a}
\end{aligned}
$$

The coefficients $\eta$ and $\nu$ in the above set of equations obtained from ensuring proper commutation relations are given by,

$$
\begin{aligned}
\eta_{k a, k^{\prime} b} & =\delta_{k, k^{\prime}} \delta_{a, b}-\frac{t_{k a} \nu_{k^{\prime} b}}{\epsilon_{k a}-\epsilon_{k^{\prime} b}+i \delta} \\
\nu_{k a} & =\frac{t_{k a}}{\epsilon_{k a}-\epsilon_{0}-\sum_{k^{\prime} b} \frac{t_{k^{\prime} b}^{2}}{\epsilon_{k a}-\epsilon_{k^{\prime} b}-i \delta}}
\end{aligned}
$$

We identify tunneling rates to the left and right leads by

$$
\Gamma_{L / R}(\epsilon)=2 \pi \sum_{k} t_{k, L / R}^{2} \delta\left(\epsilon-\epsilon_{k}\right)
$$

The current through the left lead is given by

$$
\begin{aligned}
I_{L} & =\frac{d N_{L}}{d t}=-i \frac{e}{\hbar} \sum_{k} t_{k L}\left(d^{\dagger} a_{k L}-h . c .\right) \\
\left\langle I_{L}\right\rangle & =2 \frac{e}{\hbar} \sum_{k} t_{k L} \operatorname{Im}\left\langle d^{\dagger} a_{k L}\right\rangle
\end{aligned}
$$

and charge conservation requires $I_{L}=-I_{R}$. Plugging in the expressions for $d$ and $a_{k L}$ in terms of the exact eigenstates $\alpha_{k a}$ into the expression for the current (Eq. 85) we obtain

$$
\left\langle I_{L}\right\rangle=2 \frac{e}{\hbar} \sum_{k, k_{1}, k_{2} ; \alpha, \beta=L, R} t_{L} \operatorname{Im}\left(\eta_{k L, k_{2} \beta} \nu_{k_{1}, \alpha}^{*}\left\langle\alpha_{k 1, \alpha}^{\dagger} \alpha_{k 2, \beta}\right\rangle\right)
$$

In the non-interacting limit the expectation value of the exact eigenstates is

$$
\left\langle\alpha_{k_{1} \alpha}^{\dagger} \alpha_{k_{2}, \beta}\right\rangle=\delta_{\alpha, \beta} \delta_{k_{1}, k_{2}} f\left(\epsilon_{k}-\mu_{\beta}\right)
$$

where $f(x)$ is the Fermi-Dirac distribution function. Substituting this in Eq. 86 we obtain

$$
\left\langle I_{L}\right\rangle=\frac{e}{\hbar} \int \frac{d \epsilon}{2 \pi} \frac{\Gamma_{L}(\epsilon) \Gamma_{R}(\epsilon)}{\Gamma_{L}(\epsilon)+\Gamma_{R}(\epsilon)}\left\{f\left(\epsilon-\mu_{L}\right)-f\left(\epsilon-\mu_{R}\right)\right\} A(\epsilon)
$$

where $A(\epsilon)$ is the spectral density of the dot and is given by

$$
A(\epsilon)=\frac{\Gamma_{L}(\epsilon)+\Gamma_{R}(\epsilon)}{\left(\epsilon-\epsilon_{0}-\Sigma^{\prime}(\epsilon)\right)^{2}+\left(\frac{\Gamma(\epsilon)}{2}\right)^{2}}
$$

Note that $\Sigma^{\prime}(\epsilon)$ is related to $\Gamma(\epsilon)=\Gamma_{L}(\epsilon)+\Gamma_{R}(\epsilon)$ by the usual Kramers-Kronig relation. For simplicity in our subsequent computation we assume energy independent density of states and tunneling amplitude $t_{L, R}$, so that $\Sigma^{\prime}=0$ and $\Gamma_{L, R}$ are constants. Eq. 88 agrees with the expression for the current that was derived by Wingreen et $a l .18$ employing the Keldysh non-equilibrium technique.

In the presence of electron-phonon interactions, the full Hamiltonian (now including Eq. 77 and Eq. 178) takes the following form in the basis of exact eigenstates of the noninteracting system,

$$
\begin{aligned}
H & =\sum_{k, a=L, R} \epsilon_{k} \alpha_{k, a}^{\dagger} \alpha_{k, a}+\omega_{0} b^{\dagger} b \\
& +\lambda \omega_{0}\left(b+b^{\dagger}\right) \sum_{k, k^{\prime}, a, b=L, R} \nu_{k, a}^{*} \nu_{k^{\prime}, b} \alpha_{k a}^{\dagger} \alpha_{k^{\prime} b}
\end{aligned}
$$


A standard method for studying the nonequilibrium problem posed by $H$ is to define retarded Green's function for the electrons on the dot

$$
\begin{gathered}
G_{d}^{R}\left(t, t^{\prime}\right)=-i \theta\left(t-t^{\prime}\right)\left\langle\left\{d(t), d^{\dagger}\left(t^{\prime}\right)\right\}_{+}\right\rangle \\
=-i \theta\left(t-t^{\prime}\right) \sum_{k a, k^{\prime} b} \nu_{k a} \nu_{k^{\prime} b}^{*}\left\langle\left\{\alpha_{k a}(t), \alpha_{k^{\prime} b}^{\dagger}\left(t^{\prime}\right)\right\}_{+}\right\rangle
\end{gathered}
$$

and the Keldysh Green's function for the dot electron,

$$
\begin{aligned}
G_{d}^{K}\left(t, t^{\prime}\right) & =-i\left\langle\left\{d(t), d^{\dagger}\left(t^{\prime}\right)\right\}_{-}\right\rangle \\
& =-i \sum_{k a, k^{\prime} b} \nu_{k a} \nu_{k^{\prime} b}^{*}\left\langle\left\{\alpha_{k a}(t), \alpha_{k^{\prime} b}^{\dagger}\left(t^{\prime}\right)\right\}_{-}\right\rangle
\end{aligned}
$$

Similarly for the phonons we define the corresponding retarded and Keldysh Green's functions,

$$
\begin{aligned}
& D^{R}\left(t, t^{\prime}\right)=-i \theta\left(t-t^{\prime}\right)\left\langle\left\{b(t)+b^{\dagger}(t), b\left(t^{\prime}\right)+b^{\dagger}\left(t^{\prime}\right)\right\}(9)\right. \\
& D^{K}\left(t, t^{\prime}\right)=-i\left\langle\left\{b(t)+b^{\dagger}(t), b\left(t^{\prime}\right)+b^{\dagger}\left(t^{\prime}\right)\right\}_{+}\right\rangle
\end{aligned}
$$

Note that the phonon (electron) Keldysh propagators in the equal time limit are $i D^{K}(t, t)=2\left(1+2\left\langle b^{\dagger} b\right\rangle\right)$ $\left(i G_{d}^{K}(t, t)=1-2\left\langle d^{\dagger} d\right\rangle\right)$ and are directly related to the average phonon (electron) number and therefore correspond to the first moments of the density matrix. Higher order equal time correlators $\left\langle\left(b(t) b^{\dagger}(t)\right)^{n}\right\rangle$ give higher moments of the density matrix, enabling in principle the full reduced density matrix $\rho_{D}$ to be reconstructed.

The retarded dot Green's function for a single resonant level in the absence of phonons can be easily obtained by using Eq. 87

$$
G_{d}^{R}\left(t, t^{\prime}\right)=g^{R}\left(t_{1}, t_{2}\right)=\sum_{k a}\left|\nu_{k a}\right|^{2} G_{k a, k a}^{R}\left(t_{1}, t_{2}\right)
$$

where $G_{k a, k a}^{R}\left(t, t^{\prime}\right)=-i \theta\left(t-t^{\prime}\right)\left\langle\left\{\alpha_{k a}(t), \alpha_{k a}^{\dagger}\left(t^{\prime}\right)\right\}_{+}\right\rangle=$ $-i \theta\left(t-t^{\prime}\right) e^{-i \epsilon_{k}\left(t-t^{\prime}\right)}$. It is now easy to see that in Fourier space the retarded Green's function for the noninteracting dot has the familiar form

$$
\tilde{g}^{R}(\omega)=\sum_{k a} \frac{\left|\nu_{k a}\right|^{2}}{\omega-\epsilon_{k}+i \delta}=\frac{1}{\omega-\epsilon_{0}+i \frac{\Gamma}{2}}
$$

In a similar manner the Keldysh Green's function in the absence of phonons is found to be

$$
\begin{aligned}
\tilde{g}^{K}(\omega) & =2 \pi i \sum_{k a}\left|\nu_{k a}\right|^{2} \delta\left(\omega-e_{k}\right)\left\{2 f\left(\epsilon_{k}-\mu_{a}\right)-1\right\} \\
& =-i \frac{\Gamma_{L}\left(1-2 f\left(\omega-\mu_{L}\right)\right)+\Gamma_{R}\left(1-2 f\left(\omega-\mu_{R}\right)\right)}{\left(\omega-\epsilon_{0}\right)^{2}+\frac{\Gamma^{2}}{4}}
\end{aligned}
$$

where $f(x)=1 /\left(\exp \left(\frac{x}{T}\right)+1\right)$ denotes the Fermi distribution function.

Moreover in the non-interacting limit, the retarded and Keldysh phonon Green's functions $\left(D_{0}^{R / K}\right)$ defined in Eq. 95] and 96 take the following form in Fourier space

$$
\begin{array}{r}
D_{0}^{R}(\omega)=\frac{2 \omega_{0}}{\omega^{2}-\omega_{0}^{2}+i \delta \operatorname{sgn}(\omega)}(100) \\
D_{0}^{K}(\omega)=-2 \pi i\left\{\delta\left(\omega+\omega_{0}\right)+\delta\left(\omega-\omega_{0}\right)\right\} \operatorname{coth} \frac{\omega_{0}}{2 T}(101)
\end{array}
$$

In order to calculate the current for the case when electron-phonon interactions are present, we shall use the result derived by Wingreen et al., namely that the current through an interacting dot is still given by the expression Eq. 28 but with the spectral density $A(\epsilon)=2 i \operatorname{Im}\left\{G_{d}^{R}(\epsilon)\right\}$, where $G_{d}^{R}$ is the d-electron retarded Green's function calculated under appropriate nonequilibrium conditions and with respect to the full Hamiltonian H. We shall carry out this prescription for calculating the spectral density in the next section.

\section{Keldysh Greens function method: perturbative analysis}

In the presence of non-zero electron-phonon coupling, the Dyson's equations we wish to solve may be written in the following compact form in $2 \times 2$ Keldysh space ${ }^{22}$

$$
\begin{aligned}
& \mathrm{G}_{\mathrm{d}}^{-1}=\mathrm{g}_{\mathrm{d}}^{-1}-\tilde{\boldsymbol{\Sigma}} \\
& \mathbf{D}^{-1}=\mathrm{D}_{0}^{-1}-\Pi
\end{aligned}
$$

where

$$
\mathbf{G}_{\mathbf{d}}=\left(\begin{array}{cc}
G_{d}^{R} & G_{d}^{K} \\
0 & G_{d}^{A}
\end{array}\right)
$$

is the local dot Green's function, and

$$
\tilde{\boldsymbol{\Sigma}}=\left(\begin{array}{cc}
\tilde{\Sigma}^{R} & \tilde{\Sigma}^{K} \\
0 & \tilde{\Sigma}^{A}
\end{array}\right)
$$

is the electron self-energy due to electron-phonon interactions. A similar matrix structure for the phonon propagator $\mathbf{D}$ and polarization $\boldsymbol{\Pi}$ in terms of retarded, advanced $\left(D^{R / A}, \Pi^{R, A}\right)$ and Keldysh $\left(D^{K}, \Pi^{K}\right)$ components also exists. The non-interacting Green's functions $\mathbf{g}_{\mathbf{d}}$ and $\mathbf{D}_{\mathbf{0}}$ have components that have been explicitly calculated in Eqn. 98 99 100 and 101 Note that the temperature enters explicitly via the bare electron and phonon Green's function.

We analyze the equations perturbatively. The expansion parameter is $\frac{\lambda \omega_{0}}{\Gamma}$, and the leading non-trivial $\tilde{\boldsymbol{\Sigma}}$ and $\Pi$ are represented by the diagrams in Fig. 10 We write the perturbative expansion in the usual self-energy language, but we note that in contrast to the conventional band-electron case crossed diagrams for the electron Green's function are not small relative to uncrossed diagrams, because the Green's function lacks the pole structure found in the translation invariant case. Our results for the electron Green's function and electron kinetic equation should be understood to be perturbative in $\lambda$.

To leading nontrivial order it is sufficient to calculate the phonon self-energy $\Pi \mathbf{\Pi}$ using the bare electron Green's function $\mathbf{g}_{\mathbf{d}}$, but a correct calculation of $\tilde{\boldsymbol{\Sigma}}$ requires the use of the full $\mathbf{D}$.

The retarded phonon self energy (Fig. 10) becomes

$$
\Pi^{R}\left(t, t^{\prime}\right)=-\frac{i \lambda^{2} \omega_{0}^{2}}{2}\left\{g^{R}\left(t, t^{\prime}\right) g^{K}\left(t^{\prime}, t\right)+g^{K}\left(t, t^{\prime}\right) g^{A}\left(t^{\prime}, t\right)\right\}
$$


while the Keldysh phonon self-energy is

$$
\begin{array}{cc}
\Pi^{K}\left(t, t^{\prime}\right) & =\frac{-i \lambda^{2} \omega_{0}^{2}}{2}\left\{g^{R}\left(t, t^{\prime}\right) g^{A}\left(t^{\prime}, t\right)\right. \\
\left.+g^{A}\left(t, t^{\prime}\right) g^{R}\left(t^{\prime}, t\right)+g^{K}\left(t, t^{\prime}\right) g^{K}\left(t^{\prime}, t\right)\right\}
\end{array}
$$

Going into Fourier space and using Eqns. 98 and 99 we obtain the following expression for the real and imaginary parts of $\tilde{\Pi}^{R}(\omega)$ and $\tilde{\Pi}^{K}(\omega)$,

$$
\begin{aligned}
\tilde{\Pi}_{r e}^{R}(\omega) & =-\lambda^{2}\left\{\Gamma_{L} T_{1}\left(\mu_{L}, \omega\right)+\Gamma_{L} T_{1}\left(\mu_{L},-\omega\right)(106)\right. \\
& \left.+\Gamma_{R} T_{1}\left(\mu_{R}, \omega\right)+\Gamma_{R} T_{1}\left(\mu_{R},-\omega\right)\right\} \\
\tilde{\Pi}_{i m}^{R}(\omega) & =i \lambda^{2}\left\{\Gamma_{L} T_{2}\left(\mu_{L}, \omega\right)-\Gamma_{L} T_{2}\left(\mu_{L},-\omega\right)(107)\right. \\
& \left.+\Gamma_{R} T_{2}\left(\mu_{R}, \omega\right)-\Gamma_{R} T_{2}\left(\mu_{R},-\omega\right)\right\}
\end{aligned}
$$

where we define the following integrals

$T_{1}(\mu, \omega)=\int \frac{d \omega_{2}}{2 \pi} \frac{\left(\omega+\omega_{2}-\epsilon_{0}\right)\left\{1-2 f\left(\omega_{2}-\mu\right)\right\}}{\left(\left(\omega_{2}-\epsilon_{0}\right)^{2}+\frac{\Gamma^{2}}{4}\right)\left(\left(\omega+\omega_{2}-\epsilon_{0}\right)^{2}+\frac{\Gamma^{2}}{4}\right)}$

and

$$
T_{2}(\mu, \omega)=\frac{\Gamma}{2} \int \frac{d \omega_{2}}{2 \pi} \frac{1-2 f\left(\omega_{2}-\mu\right)}{\left(\left(\omega_{2}-\epsilon_{0}\right)^{2}+\frac{\Gamma^{2}}{4}\right)\left(\left(\omega+\omega_{2}-\epsilon_{0}\right)^{2}+\frac{\Gamma^{2}}{4}\right)}
$$

Analytic expressions for $T_{1}(\mu, \omega)$ and $T_{2}(\mu, \omega)$ may be obtained at zero temperature, and are given in Appendix A.

Note that the combination $T_{1}\left(\mu_{L}, \Gamma_{L}, \omega\right)+$ $T_{1}\left(\mu_{L}, \Gamma_{L},-\omega\right)$ is symmetric, while $T_{2}\left(\mu_{L}, \Gamma_{L}, \omega\right)-$ $T_{2}\left(\mu_{L}, \Gamma_{L},-\omega\right)$ is asymmetric with respect to $\omega$. As a result, for all combination of couplings and applied voltages

$$
\begin{array}{r}
\tilde{\Pi}_{r e}^{R}(-\omega)=\tilde{\Pi}_{r e}^{R}(\omega) \\
\tilde{\Pi}_{i m}^{R}(-\omega)=-\tilde{\Pi}_{i m}^{R}(\omega) \\
\tilde{\Pi}^{K}(-\omega)=\tilde{\Pi}^{K}(\omega)
\end{array}
$$

The retarded electron self-energy represented by the diagram in Fig. 10 is

$$
\Sigma^{R}\left(t, t^{\prime}\right)=\frac{i \lambda^{2} \omega_{0}^{2}}{2}\left\{g^{R}\left(t, t^{\prime}\right) D^{K}\left(t, t^{\prime}\right)+g^{K}\left(t, t^{\prime}\right) D^{R}\left(t, t^{\prime}\right)\right\}
$$

Once we know the components of the polarization matrix $\boldsymbol{\Pi}$, we may use the Dyson equations 103 together with the following parameterization for $D^{K 23}$

$$
D^{K}=D^{R} \hat{f}_{k}^{p h}-\hat{f}_{k}^{p h} D^{A}
$$
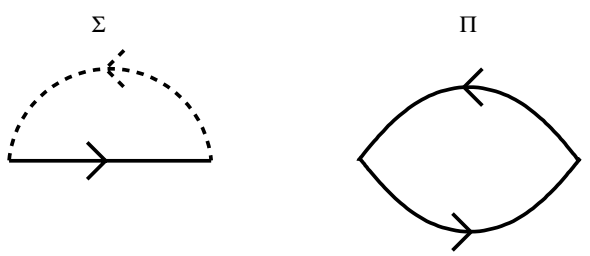

FIG. 10: Diagrams that correspond to the leading contribution to the electron self-energy $(\Sigma)$ and the phonon polarization (П).

to obtain,

$$
\begin{aligned}
D^{R}(\omega)=\frac{2 \omega_{0}}{\omega^{2}-\omega_{0}^{2}+i \delta \operatorname{sgn}(\omega)}-2 \omega_{0} \Pi^{R}(\omega) & \\
\left(i \frac{\partial}{\partial t_{1}}+i \frac{\partial}{\partial t_{2}}\right) f_{k}^{p h}\left(t_{1}, t_{2}\right) & =\Pi^{R} f_{k}^{p h} \\
& -f_{k}^{p h} \Pi^{A}-\Pi^{K}
\end{aligned}
$$

Note that our parameterization in terms of $f_{k}^{p h}$ is such that at equilibrium $f_{k}^{p h}(x)=\operatorname{coth}(x)=1+2 n_{B}(x), n_{B}$ being the Bose distribution function.

Besides $\Sigma^{R}$ it is also useful to evaluate the Keldysh component of the self-energy $\Sigma^{K}$ that we will later use in order to calculate the distribution function of the dot. To the perturbative order to which we work, $\Sigma^{K}$ is related to the non-interacting Green's function of the dot and the phonon Green's function as follows

$$
\begin{aligned}
2 \frac{\sum^{K}\left(t, t^{\prime}\right)}{i \lambda^{2} \omega_{0}^{2}} & =g^{R}\left(t, t^{\prime}\right) D^{R}\left(t, t^{\prime}\right) \\
& +g^{A}\left(t, t^{\prime}\right) D^{A}\left(t, t^{\prime}\right)+g^{K}\left(t, t^{\prime}\right) D^{K}\left(t, t^{\prime}\right)
\end{aligned}
$$

Now that we know the retarded electron self-energy, we can use Eq. 88 to calculate the current which is given by,

$$
\begin{gathered}
I\left(\mu_{L}, \mu_{R}\right)=\frac{e^{2}}{\hbar}\left(\frac{\Gamma_{L} \Gamma_{R}}{\Gamma_{L}+\Gamma_{R}}\right) \int \frac{d \omega}{2 \pi}\left(f\left(\omega-\mu_{L}\right)-f\left(\omega-\mu_{R}\right)\right) \\
\frac{\Gamma-2 \tilde{\Sigma}_{i m}^{R}\left(\omega, \mu_{L}, \mu_{R}\right)}{\left(\omega-\epsilon_{0}-\tilde{\Sigma}_{r e}^{R}\left(\omega, \mu_{L}, \mu_{R}\right)\right)^{2}+\left(\frac{\Gamma}{2}-\tilde{\Sigma}_{i m}^{R}\left(\omega, \mu_{L}, \mu_{R}\right)\right)^{2}}
\end{gathered}
$$




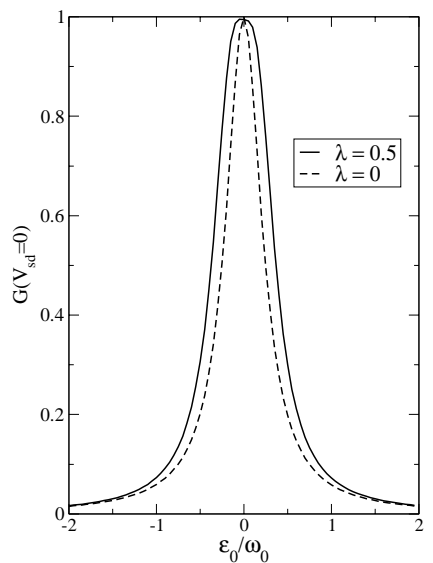

FIG. 11: Zero bias conductance (in units of $\frac{e^{2}}{h}$ ) as a function of level energy (or gate voltage) with $\left(\omega_{0}=2 \Gamma\right.$, solid line) and without coupling to a vibrational mode (dotted line).

In the next two subsections we present our results for the two extreme cases of phonons strongly coupled to a heat bath, and therefore always in equilibrium, and of phonons uncoupled to an external environment.

\section{Results : Equilibrated phonons}

In this sub-section we specialize to the case where the phonons are always in their ground state so that the phonon Green's functions $D^{R}$ and $D^{K}$ in equations 114 and 118 are always calculated under the condition that $\mu_{L}=\mu_{R}$, while all the non-equilibrium effects are included in the electron Green's functions. An important property of $\Sigma_{i m}^{R}(\omega)$ is that at equilibrium it is zero for $\omega=0$, and from this and Eq. 119 it immediately follows that the zero bias conductance even in the presence of electron-phonon coupling has the form

$$
G(V=0)=\frac{e^{2}}{h} \frac{4 \Gamma_{L} \Gamma_{R}}{\left(\Gamma_{L}+\Gamma_{R}\right)^{2}}
$$

for the case where the two lead chemical potentials in addition to being equal to each other are also aligned with the dot level $\epsilon_{d}$. Fig. 11] shows the gate voltage dependence of the zero bias conductance for symmetric broadening and two values of $\lambda$.

Fig. 12 presents our result for the current (conductance) for the equilibrated phonon case at zero temperature. The top panel is the difference between the current with and without electron-phonon coupling for two different bias conditions. Note that for asymmetrically applied biases, the current with phonons can take a value larger than that in the absence of phonons. This is due to a shift in the center position of the spectral density (the total area under the spectral density being conserved). The

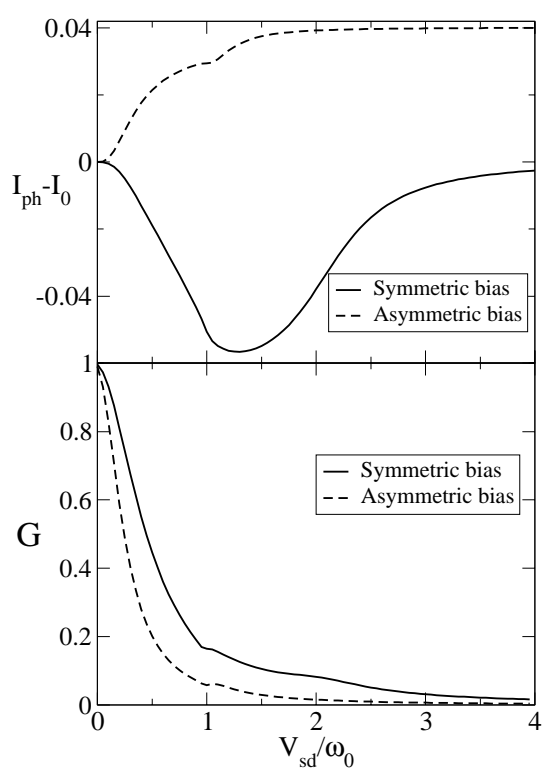

FIG. 12: Zero temperature current (in units of $\frac{e \Gamma}{h}$ and measured relative to no phonon current $I_{0}$ [upper panel] ) and conductance [lower panel] (in units of $\frac{e^{2}}{h}$ ) under asymmetric $\left(\mu_{R}=0, \mu_{L}=V_{s d}\right)$ and symmetric $\left(\mu_{L}=-\mu_{R}=V_{s d} / 2\right)$ bias conditions and for equilibrated phonons. The phonon frequency $\omega_{0}=2.0 \Gamma$ and $\Gamma_{L}=\Gamma_{R}=0.5 \Gamma$. Moreover the electron-phonon dimensionless coupling strength is $\lambda=0.25$. Note that under conditions of asymmetric bias, the current can saturate to a value larger than that for the device without phonons $(\lambda=0)$.

lower panel is conductance for the same bias conditions. The conductance for the symmetric bias $\left(\mu_{L}=-\mu_{R}\right)$ case shows two features, one at $V_{s d} / \omega_{0}=1.0$ and the other is a much broader feature at $V_{s d} / \omega_{0}=2.0$. While the former corresponds to the onset of in-elastic cotunneling, the latter corresponds to the onset of sequential tunneling. (Under the asymmetric bias condition of $\mu_{L}=V_{s d}, \mu_{R}=0$ one observes only sequential tunneling).

The transition from cotunneling dominant current to sequential tunneling dominant current can be understood by studying how the imaginary part of the electron selfenergy due to interaction with phonons $\left(\tilde{\Sigma}_{i m}^{R}\right)$ evolves with bias. Fig. 13 shows $\tilde{\Sigma}_{i m}^{R}(\omega)$ for the symmetric bias case $\mu_{L}=V_{s d} / 2=-\mu_{R}=\omega_{0} / 2$ and for two different values of $\Gamma / \omega_{0}$. For simplicity we have considered the case where $\Gamma_{L}=\Gamma_{R}$. Under symmetric bias, $\tilde{\Sigma}_{i m}^{R}$ increases rapidly for $|\omega|>\left|\omega_{0}-V_{s d} / 2\right|$, while in calculating the current the spectral density is integrated from $-V_{s d} / 2$ to $V_{s d} / 2$ (see Eq. 119). Therefore clearly there is a threshold at $V_{s d}=\omega_{0}$ when the $\tilde{\Sigma}_{i m}\left(\omega=\omega_{0} / 2\right)$ jumps by $\propto \frac{\Gamma}{\omega_{0}^{2}+\Gamma^{2}}$, and this corresponds to the onset of inelastic cotunneling. As the voltage is increased further, the range of integration also increases to finally include the Lorentizian broadening centered around $\omega=\omega_{0}$, and this 


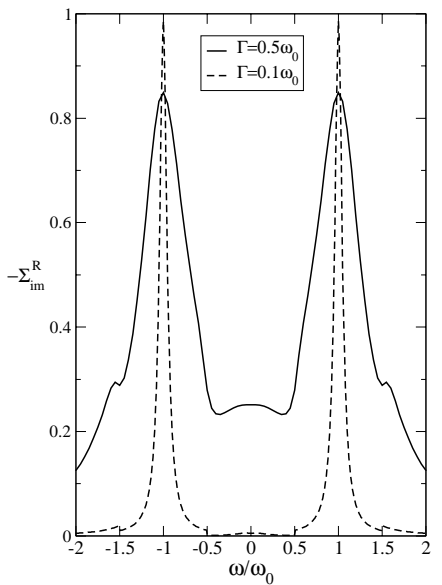

FIG. 13: Imaginary part of the electron self-energy (in units of $\Gamma$ ) due to phonons for the symmetric bias condition $\mu_{L}=$ $-\mu_{R}=V_{s d} / 2=\omega_{0} / 2$, and $\lambda \omega_{0}=1 \Gamma$, level energy $\epsilon_{0}=0$.

corresponds to the onset of sequential tunneling.

As $\Gamma$ is made smaller, the size of the step in $\tilde{\Sigma}_{i m}^{R}(\omega=$ $\left.\omega_{0} / 2\right)$ decreases, thus decreasing the cotunneling contribution to the conductance. Moreover the lorentzian broadening in the self-energy around $\omega_{0}$ also becomes narrower, so that the sequential tunneling peak in the conductance also gets sharper.

Fig. 14] shows the temperature dependence of inelastic cotunneling under conditions of no coupling to a heat bath (unequilibrated phonons). Calculations for this have been performed in the regime $\epsilon_{0}>\mu_{L}, \mu_{R}$ so that the current is entirely due to cotunneling. (The current under resonant conditions for this unequilibrated phonon case is discussed in detail in the next section). It is clear from Fig. 14that inelastic cotunneling shows up as a step in $d I / d V$ that gets rounded very rapidly with increasing temperature.

\section{E. Results: Unequilibrated phonons}

For out of equilibrium conditions, we may derive a quantum Boltzmann equation for the mean phonon number, which for weak electron-phonon couplings is identified as $\left\langle N_{p h}\right\rangle=\frac{-1+f_{k}^{p h}\left(\omega=\omega_{0}\right)}{2}$. (This is from using Eq. 115] and the fact that for weak couplings $D_{i m}^{R}$ is almost a delta function at the phonon frequency). Therefore Eq. 117 rewritten under steady state conditions and

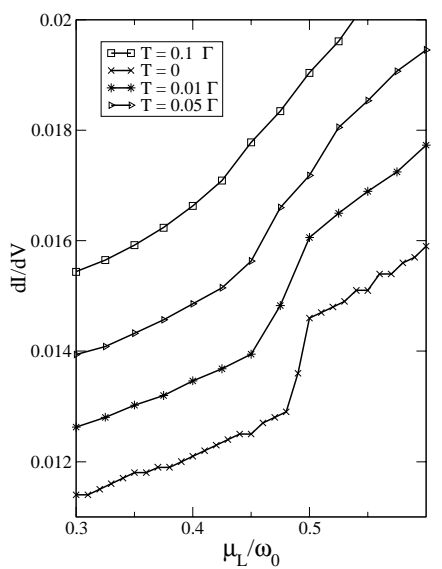

FIG. 14: Cotunneling $\mathrm{dI} / \mathrm{dV}$ (in units of $\frac{e^{2}}{\hbar}$ ) for bias conditions $\mu_{L}=-\mu_{R}=V_{s d} / 2$, level energy $\epsilon_{0}=4.0 \Gamma, \omega_{0}=2 \Gamma$, $\lambda \omega_{0}=1 \Gamma$ and for phonons not coupled to any heat bath.

at an on-shell frequency has the form

$$
\begin{aligned}
0 & =\left\langle N_{p h}\right\rangle\left(\Pi^{K}\left(\omega_{0}\right)+\Pi^{R}\left(\omega_{0}\right)-\Pi^{A}\left(\omega_{0}\right)\right) \\
& -\left(1+\left\langle N_{p h}\right\rangle\right)\left(\Pi^{K}\left(\omega_{0}\right)-\left(\Pi^{R}\left(\omega_{0}\right)-\Pi^{A}\left(\omega_{0}\right)\right)\right)
\end{aligned}
$$

From this the phonon outscattering rate may be identified as $\left\{\Pi^{K}\left(\omega_{0}\right)+\Pi^{R}\left(\omega_{0}\right)-\Pi^{A}\left(\omega_{0}\right)\right\}$, while the inscattering rate may be identified as $\left\{\Pi^{K}\left(\omega_{0}\right)-\left(\Pi^{R}\left(\omega_{0}\right)-\right.\right.$ $\left.\left.\Pi^{A}\left(\omega_{0}\right)\right)\right\} \cdot\left\langle N_{p h}\right\rangle$ is plotted in Fig. [15 for a variety of bias conditions. The results here are similar to what was observed in the high- $T$ classical calculation, namely that for bias conditions under which the dot is half filled or close to it, the phonons tend to go far out of equilibrium. When the phonons deviate considerably from their ground state, the corrections to the electron-self energy become comparable to $\Gamma$ and one is no longer within the perturbative regime. Therefore the results for the current and conductance that we present here (Fig. 16) are for the case of $\mu_{L}=V_{s d}, \mu_{R}=0.0$, when the phonons acquire a steady state distribution at large biases that is not far from its equilibrium value and a perturbative approximation is valid.

The upper panel of Fig. 16plots the difference between the current with and without phonons for the asymmetric bias case, and for comparision this is plotted for both equilibrated and unequilibrated phonons, while the lower panel is the conductance peak corresponding to the first phonon side-band. Again within perturbation theory the differences between these two cases is not significant. 


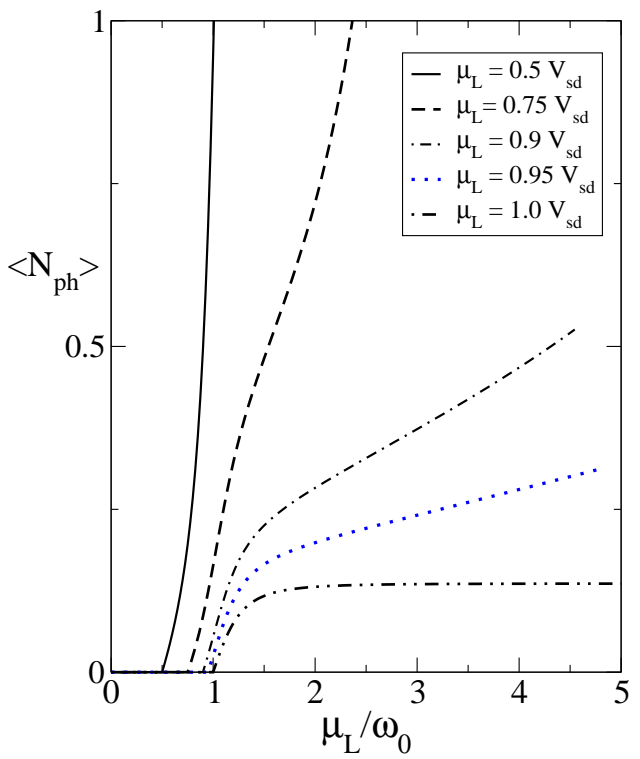

FIG. 15: Plot of average phonon occupation number for $\omega_{0}=$ $2.0 \Gamma$, for several different bias conditions. Except for the most asymmetric case $\left(\mu_{L}=V_{s d}\right)$, the phonon number diverges for large enough bias voltages. Also note that the onset voltage for the deviation of the phonon number from its equilibrium value (of zero) also continously shifts from $\mu_{L}=\omega_{0}$ (most asymmetric bias) to $\mu_{L}=\omega_{0} / 2$ (symmetric bias) signaling inelastic cotunneling.

\section{F. Perturbative calculation for Phonon distribution function}

So far the out-of equilibrium Green's function technique allowed us to calculate various averages such as current and mean phonon number. However it is also interesting to ask what the phonon probability distribution function itself is under non-equilibrium steady state conditions. In order to do so we revert to the density matrix formalism developed in Section II. The complication in calculating this quantity is the nontrivial form of the operator equation Eq. 19] One can however carry out the calculation for the rates to leading order in the electronphonon coupling, using the diagrammatic language developed by various authors 24.25 . In implementing this we again find it convenient to be in the exact eigenstate basis for the non-interacting system (Eq. 90). The leading order contribution to the rates is obtained by expanding the exponentials entering in the quantum rate equation 19 to leading order in the electron phonon coupling. The explicit form for the in-scattering and outscattering rates is therefore

$$
\begin{gathered}
R_{n \rightarrow n \pm 1}=\operatorname{Tr}_{\text {leads }} \int_{-\infty}^{0} d t\left\langle n\left|H_{e-p h}(0)\right| n \pm 1\right\rangle \\
\left\langle n \pm 1\left|H_{e-p h}(t)\right| n\right\rangle
\end{gathered}
$$

where

$$
H_{e-p h}(t)=\lambda \omega_{0}\left(b(t)+b^{\dagger}(t)\right) \sum_{k, k^{\prime}, a, b=L, R} \nu_{k, a}^{*} \nu_{k^{\prime}, b} \alpha_{k a}^{\dagger}(t) \alpha_{k^{\prime} b}(t)
$$

On evaluating the above expressions we obtain the following quantum rate equation for the distribution function

$$
\begin{aligned}
0 & =-P_{n}^{p h}\left(R_{n \rightarrow n+1}+R_{n \rightarrow n-1}\right) \\
& +P_{n+1}^{p h} R_{n+1 \rightarrow n}+P_{n-1}^{p h} R_{n-1 \rightarrow n}
\end{aligned}
$$

where the in-scattering rate is given by

$$
\begin{gathered}
R_{n \rightarrow n+1}=\lambda^{2} \omega_{0}^{2}(n+1) \int \frac{d \epsilon}{4 \pi} \\
\frac{\sum_{a, b=L, R} \Gamma_{a} \Gamma_{b} f\left(\epsilon-\mu_{a}\right)\left(1-f\left(\epsilon-\omega_{0}-\mu_{b}\right)\right)}{\left\{\left(\epsilon-\epsilon_{0}\right)^{2}+\frac{\Gamma^{2}}{4}\right\}\left\{\left(\epsilon-\epsilon_{0}-\omega_{0}\right)^{2}+\frac{\Gamma^{2}}{4}\right\}}=(n+1) r_{i n}
\end{gathered}
$$

and the out-scattering rate is given by

$$
\begin{gathered}
R_{n \rightarrow n-1}=\lambda^{2} \omega_{0}^{2} n \int \frac{d \epsilon}{4 \pi} \\
\frac{\sum_{a, b=L, R} \Gamma_{a} \Gamma_{b} f\left(\epsilon-\mu_{a}\right)\left(1-f\left(\epsilon+\omega_{0}-\mu_{b}\right)\right)}{\left\{\left(\epsilon-\epsilon_{0}\right)^{2}+\frac{\Gamma^{2}}{4}\right\}\left\{\left(\epsilon-\epsilon_{0}+\omega_{0}\right)^{2}+\frac{\Gamma^{2}}{4}\right\}}=n r_{\text {out }}
\end{gathered}
$$

We have used short hand notations $r_{\text {in/out }}$ for the cumbersome integrals that appear in the definition of $R_{n \rightarrow n+1}$ etc. In terms of $r_{\text {in/out }}$ the quantum Boltzmann equation 124 takes the form,

$$
P_{n}^{p h}\left\{(n+1) \frac{r_{\text {in }}}{r_{\text {out }}}+n\right\}=P_{n+1}^{p h}(n+1)+(n) P_{n-1}^{p h} \frac{r_{\text {in }}}{r_{\text {out }}}
$$

It is easy to check that the above equation has a simple solution given by

$$
P_{n}^{p h}=\left(1-\frac{r_{\text {in }}}{r_{\text {out }}}\right)\left(\frac{r_{\text {in }}}{r_{\text {out }}}\right)^{n}
$$

The quantity $\frac{r_{\text {in }}}{r_{\text {out }}}$ has been plotted for various combinations of $\Gamma$ and $\omega_{0}$ in Fig. 17 The figure illustrates that the rapidity with which the phonon distribution diverges with bias depends on the relative sizes of $\Gamma$ and $\omega_{0}$. The stronger the coupling to the leads, the more easily the phonons equilibrate.

In the high- $T$ classical regime the rate equations for phonons for weak electron-phonon coupling has the same structure as Eq. [127 but with modified scattering rates $r_{\text {in/out }}$. (This has been explicitly shown in Appendix B). It therefore follows that even in the high- $\mathrm{T}$ regime, the phonon distribution function is given by Eq. 128

Note that on comparing Eqns. 1261125 with Eqns. 107108 we find, not surprisingly,

$$
\frac{r_{\text {in }}}{r_{\text {out }}}=\left.\frac{\Pi^{K}-\left(\Pi^{R}-\Pi^{A}\right)}{\Pi^{K}+\Pi^{R}-\Pi^{A}}\right|_{\omega=\omega_{0}}
$$

where the polarization $\Pi$ were evaluated within the Keldysh Green's function approach. Thus in particular $\left\langle N_{p h}\right\rangle=\frac{-D_{K}(t=0)+1}{2}=\sum_{n} n P_{n}^{p h}$, where $D_{K}$ has been calculated for phonons with no life-time broadening. 


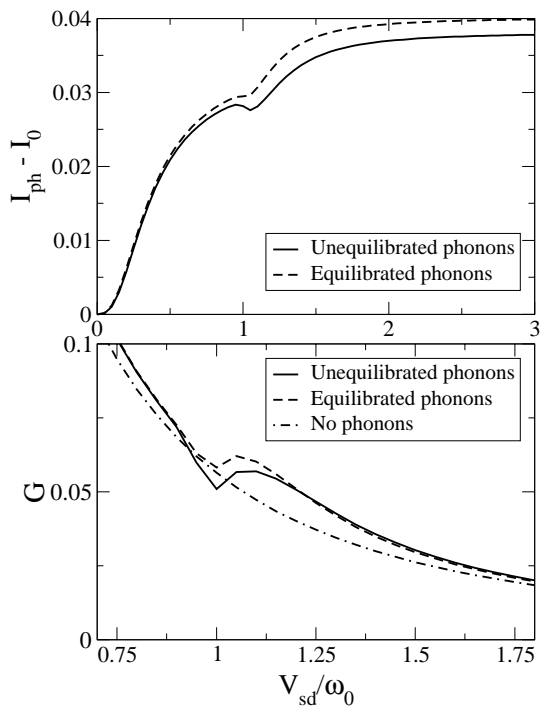

FIG. 16: Zero temperature conductance and current for equilibrated, unequilibrated and non-interacting levels under asymmetric bias conditions $\left(\mu_{R}=0, \mu_{L}=V_{s d}\right)$. The phonon frequency $\omega_{0}=2.0 \Gamma$ and $\Gamma_{L}=\Gamma_{R}=0.5 \Gamma$. Moreover the electron-phonon dimensionless coupling strength is $\lambda=0.25$. Note that $I$ (upper panel) is in units of $\frac{e \Gamma}{h}$ and $G$ (lower panel) is in units of $\frac{e^{2}}{h}$.

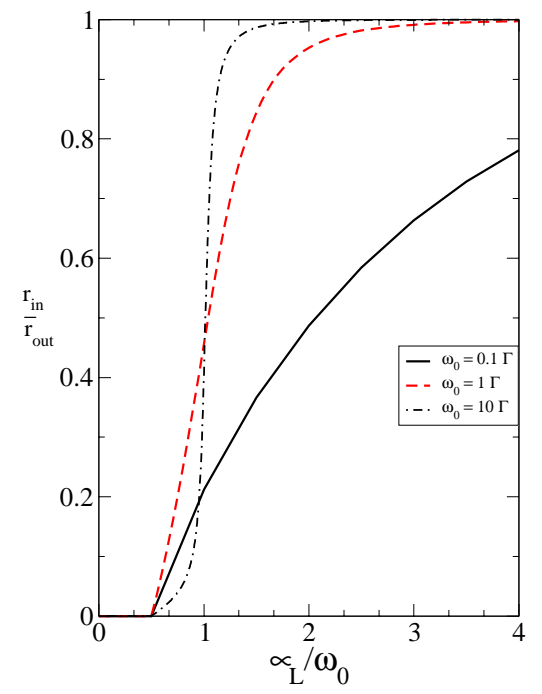

FIG. 17: Plot of ratio of phonon inscattering and outscattering rates $\frac{r_{i n}}{r_{0 u}}$ for several different ratios of $\omega_{0} / \Gamma$. The bias conditions are $\mu_{L}=-\mu_{R}$, and the onset of a non-zero $r_{i n}$ at $\mu_{L}=\omega_{0} / 2$ is the sign of inelastic cotunneling. The high-T limit is approached for $\Gamma \ll \omega_{0}$, when the onset of nonzero $r_{i n}$ shifts to the sequential tunneling limit of $\mu_{L}=\omega_{0}$.

\section{LOW T TO HIGH-T CROSSOVER}

We would now like to connect our low-T quantum calculation with the high-T calculation discussed in Section III. The high-T limit may be reached by taking $T \gg \Gamma$ in the Keldysh calculation, and the crossover from the $T=0$ to $T \gg \Gamma$ case has been illustrated in figures 18 and 19 for equilibrated and unequilibrated phonons respectively. The results for the equilibrated phonons have been presented for a rather large electron-phonon coupling $\lambda \omega_{0}=10 \Gamma$ which strictly speaking is beyond the limits of validity of the perturbative approximation used here, in order to illustrate how the phonon sidebands evolve with temperature.

The top panel of Fig. 18]illustrates how the elastic resonance broaden with temperature, while the lower panel shows the broadening of the phonon side-band. As is evident from the two panels, at high temperatures the agreement between the high- $\mathrm{T}$ rate equation calculation and the quantum calculation is much better for the case of phonons with no life-time broadening. The effect of the life-time broadening due to interactions with electrons is to round off the phonon side-band further.

Fig. 19 illustrates the cross-over from the low- $\mathrm{T}$ to high-T regime under conditions of unequilibrated phonons and within the perturbative limit of $\lambda \omega_{0}=0.5 \Gamma$ and asymmetric bias. The phonon side-bands vanish for $T>\lambda \omega_{0}$.

\section{CONCLUSIONS}

\section{A. Summary}

In this paper we have studied a simple model of an electron-phonon coupled quantum dot, involving a (possibly degenerate) electron level coupled to leads and to phonons. The problem has four different important "internal" parameters: a dimensionless electron-phonon coupling $\lambda$ (defined for example in Eq. 22), the ratio of the phonon frequency $\omega_{0}$ to the broadening $\Gamma$ of the on-site level due to coupling to the leads, the ratio of the temperature $T$ to the level width $\Gamma$. (A fourth parameter, the ratio of the rate $\gamma_{e q}$ at which the phonons relax to the heat bath characteristic of the device, to the mean electron current flow rate has only been studied in limiting cases).

The model admits two important sub-cases: of phonons coupled to the number of electrons on the molecule (Eq. 2), and of phonons coupled to the dotlead hybridization (Eq. (4). Our formulation applies to both cases, but we have focussed mostly on the former (McCarthy et al. have considered special features of the latter case in the classical regime). In this paper we have attempted to present a general framework, within which different special cases can be analyzed as desired. There are two important crossovers: the electronic quantal-classical crossover controlled by $T / \Gamma$, and 

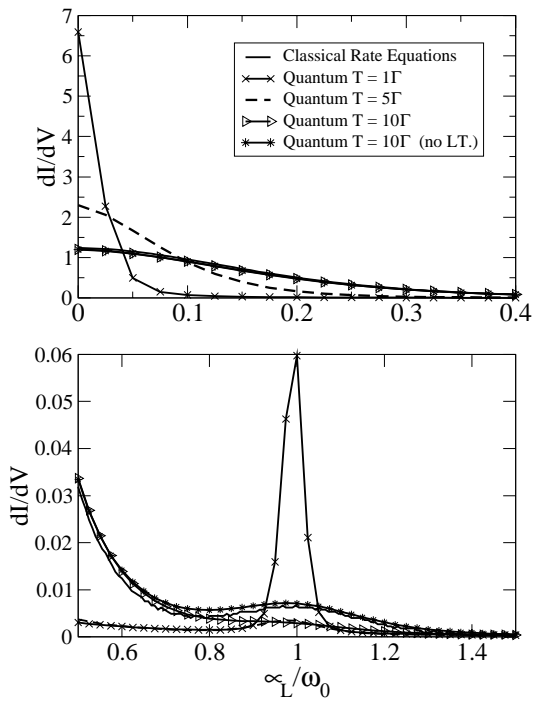

FIG. 18: Quantal-classical crossover effects in differential conductance spectra for equilibrated phonons. Upper panel: temperature dependence of zero bias "resonance" peak computed as described in text and compared to results obtained from classical rate equations (section III, but $U=0$ ). Lower panel: temperature dependence of first phonon sideband, computed as described in text and compared to results obtained from classical rate equations. The rapid thermal smearing of both central peak and phonon sideband is evident. Note that in the phonon side-band case, broadening of the phonon level due to electron phonon coupling leads to additional smearing not included in the rate equation model. The parameters are $\omega_{0}=100 \Gamma, \lambda=0.1$ and $T=1,5,10 \Gamma$. The bias conditions are $\mu_{L}=-\mu_{R}$. Rate equation calculation: $\lambda=0.1, T=10 \Gamma$. Note: $d I / d V$ is in units of $\frac{e^{2}}{\hbar} \frac{\Gamma}{\omega_{0}}$.

the phonon adiabatic/anti-adiabatic (phonon frequency long or short relative to inverse electron dwell time on molecule) crossover controlled by $\omega_{0} / \Gamma$. (The limit of $\omega_{0} \ll T$ is not interesting). In the classical limit (roughly, $T / \Gamma>1$ ) this program has been carried out completely for all values of coupling $\lambda$ by us and by other workers. The relation between our results and those of other workers is discussed in detail in subsection B below.

The model has two external "control" parameters; the source-drain voltage difference $V_{s d}=\mu_{L}-\mu_{R}$ (see Fig. 1) and the molecule one-electron addition energy $\epsilon^{\prime}$ measured relative to the average of the source-drain voltage $\left(\mu_{L}+\mu_{R}\right) / 2$, and also referred to as the gate voltage. It is well known that the conductance exhibits steps whenever one of the lead chemical potentials passes through the level energy $\epsilon^{\prime}$. The important new consequence of electron-phonon coupling is the appearance of steps such as those shown in Fig. B in the IV characteristics, when the source-drain voltage passes though an integer multiple of the phonon frequency. The existence of the phonon

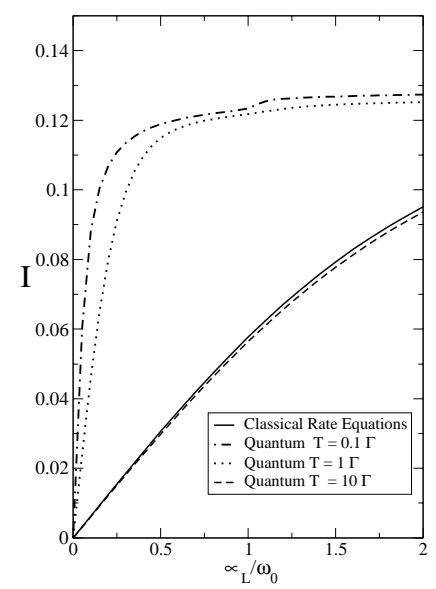

FIG. 19: Quantal to classical crossover effects on IV curve for unequilibrated phonons. The parameters are $\omega_{0}=10 \Gamma$, $\lambda=0.05$ and the temperature for the classical calculation corresponds to $T=10.0 \Gamma$. The quantum calculation has been performed for $T=0.1,1,10 \Gamma$. The results are for asymmetric bias $\mu_{R}=0.0, \mu_{L}=V_{s d}$ when the perturbation approximation is valid. Note: $I$ is in units of $\frac{e \Gamma}{\hbar}$.

sidebands has been noted by several authors 7.8 . An important new result (Fig. 4) of our work is the systematic study of the dependence of the phonon heights on whether the phonon distribution function is controlled by the nonequilibrium current or is equilibrated to a heat bath (Aji et al. presented similar information for the cotunneling regime). We have also studied (Figs. 56 15) the non-equilibrium phonon distribution for different bias voltages. A further new result of our work is the theory for noise in the classical limit (section III C) showing that noise is a powerful spectroscopy of the degree to which the phonons are equilibrated (Fig. 8] 9), especially in the weak coupling limit.

In the quantal limit $(T<\Gamma)$, our treatment is restricted to low orders of perturbation theory in the coupling constant. Within this approximation we are able to obtain results (Figs 14] 16, 18 for the I-V characteristics including both the "direct tunneling" $\left(\mu_{L}>\epsilon^{\prime}>\mu_{R}\right.$ or conversely) and "cotunneling" regimes $\left(\mu_{L}, \mu_{R}>\epsilon^{\prime}\right.$ or conversely) and were able to treat the quantal to classical crossover (Figs 17, 18, 19). We presented both a diagrammatic (Keldysh) calculation and a solution based on the construction of exact eigenstates, and confirmed that the peculiar broadening of the phonon distribution found in the "unequilibrated classical" case survives also in the quantum limit.

\section{B. Other work}

In this section we consider the relation of our results to the extensive existing theoretical literature. The sub- 
ject was pioneered by Glazman and Shekter ${ }^{7}$ who showed that the problem of a single electron transiting a resonant level and coupled to phonons can be solved in complete analytic detail. They determined the form of the phonon side-bands in the transmission amplitude and showed how the resonant behaviour was modified by the electron-phonon interaction. Very similar results were subsequently obtained by Wingreen, Jacobsen and Wilkins ${ }^{8}$. However the single electron approximation used in these papers is not applicable to the case of leads containing a Fermi sea of electrons. The presence of other electrons blocks some of the intermediate states in the electron- phonon scattering process, changing the form of the eigenstates and crucially blocks some of the final states in the transmission process. This blocking ensures that the $T=0$ linear response conductance at resonance takes the quantized value if $2 e^{2} / h$, whereas the on resonance transmission probabilities calculated in refs 7.8 are less than unity. This issue was very recently also discussed by Flensberg ${ }^{15}$.

One crucial consequence of the presence of Fermi seas in the leads is the "floating" of the phonon side-bands in the electron spectral function and thus in the IV curve. At $V_{s d}=0$, the features in the spectral function occur at energies displaced from the fermi-level by integer multiples of the phonon frequency, thus the corresponding steps in the IV curve are observed when $V_{s d}$ is swept, but are not observed if $V_{g}$ (i.e., the mean lead Fermi level) is changed at fixed small $V_{s d}$ (see Fig. 11). Several authors ${ }^{9.10 .14}$ employ approximations to the electron Green's function corresponding roughly to those of Glazman and Shekhter or Wingreen and coworkers. These miss the above physics and erroneously predicts phonon side-bands as $V_{g}$ is varied ${ }^{9.10}$.

As also noted by Flensberg the approximations employed by refs $\frac{9,10}{}$ amount to writing the dot Green's function as $G_{d}^{R}(t)=e^{\left(i \epsilon_{0}-\Gamma\right) t}\left\langle X^{\dagger}(t) X(0)\right\rangle$ (where the operators $X$ have been defined in Eq. 31). This approximation becomes exact in the high temperature limit, and can be used as the starting point of an alternative derivation of the rate equations. However some of the literature ${ }^{14}$ who have used this approach have treated the X-operator matrix elements in an approximate manner which does not capture the structure giving rise to the step height variations displayed in our Fig. 4

Finally Gogolin and Komnik have used an adiabatic (slow phonons, fast electrons) semiclassical approximation to explore the strongly electron-phonon coupled regime. It is well known that at strong coupling the energy as a function of phonon coordinate become bistable, signaling the onset of polaronic instability. Ref ${ }^{13}$ considered the behaviour of the polaronic state under nonequilibrium conditions, and observed that a bistable I-V characteristic could result. By contrast our rate equation analysis always leads to single valued I-V curves. The bistability corresponds to a first-order "energy landscape"; however the system under study is zero dimensional, and (at least within the approach used here) ther- mal and quantal fluctuations allow the system to explore all of phase space wiping out any phase transition behaviour. The calculation of Gogolin and Komnik are presented for a different regime $(T \ll \Gamma)$, but we suspect that fluctuations would also eliminate the apparent transition in that case. An enhanced low frequency noise might however occur.

Four recent paper have appeared which present results consistent with those presented here, but emphasizing somewhat different aspects of the physics. Mc Carthy et al ${ }^{11}$ used the rate equation approach of our section III, our results reproduce theirs; however the main interest if ref 11 was in the IV curves of phonons coupled to the dot-lead hybridization and the principal focus was on thermally equilibrated phonons. Our result extend theirs by treating the nonequilibrium case (see eg. Fig. 51) and the noise (see eg. Fig. 9).

Fedorets and coworkers ${ }^{16}$ have analyzed the same problem as McCarthy et al. but in the limit of very weak coupling of phonons to a heat bath. They find that an instability occurs for $V_{s d}$ greater than a critical value. Interestingly in the weak coupling limit the critical $V_{s d}$ reported is identical to the critical $V_{s d}$ found in our calculation above which the phonon distribution become broad (see Fig. 5). According to the refs 16 the instability occurs if one has both lead coupled and dot coupled phonons, whereas the broad distribution is generic. However, this relation deserves further exploration. Here we observe that the calculations of refs ${ }^{16}$ (as well as those of our Section III) are based on a sequential-tunneling approximation. In this approximation when the threshold is exceeded the distribution we find changes rapidly (in the weak electron-phonon coupling limit) from very narrow to very broad. However if cotunneling processes (in particular the electron contribution to the phonon lifetime) are included, the transition becomes broadened with the onset moving to a lower voltage (see Fig. 15). We find the breadth of the distribution to depend strongly on the bias asymmetry, i.e., to the average occupation of the dot.

Flensberg ${ }^{15}$ has used an approximate equation of motion approach to analyze the quantum limit, determining in particular the equilibrium dot spectral function and presenting a clear analysis of the terms omitted in previous approaches 7.8 . Where there is overlap our results are in agreement with his, however non-equilibrium conditions have not been considered in this reference ${ }^{15}$.

Finally Aji, Moore and Varma 12 have considered phonon effects on the cotunneling spectrum. Their results are in essential agreement with ours, however we note that the steps in $d I / d V$ which they report to be vertical at $T=0$ are in fact smoothed by the phonon lifetime arising from electron-phonon coupling (see Fig. 14) or to coupling to a heat bath. Also we find that the steps are thermally broadened into indetectability at a relatively low $\mathrm{T}$ fixed by the step height and the phonon lifetime induced slope, rather than generically visible up to temperatures of order the phonon frequency, as stated 
in the reference ${ }^{12}$.

As far as experiments are concerned, three recent papers ${ }^{4.5 .6}$ have observed signatures in the current-voltage characteristics which are attributed to coupling to a molecular vibrational mode. A direct comparison to our results is not yet feasible because noise measurements have not been performed, and most of the samples studied show one or at most two phonon sidebands. (Only one of the samples of Ref $\frac{5}{5}$ was reported to show more).

\section{Future Directions}

Finally we briefly mention a few issues raised by this work, but not resolved. One important research area is the extension of our quantum limit results beyond the perturbative regime (in particular to the nonequilibrium polaron limit), a second is to obtain the frequency dependence of the noise spectra in the classical and quantal regimes, a third issue is the crossover between equilibrated and unequilibrated phonons, and a fourth issue is to explore the connection between the nonequilibrium distributions we find and the instabilities discussed by the Chalmers group ${ }^{16}$. Another interesting question is to explore the effect of image charges induced in the surrounding electrodes on $\mathrm{I}-\mathrm{V}^{26}$ which have been argued to be important in certain experiments ${ }^{6}$. Work in these directions is in progress.

Acknowledgements: The authors would like to thank L. Glazman and K. Matveev for helpful conversations. We acknowledge support from NSF-DMR-0338376 and Columbia University, and AJM thanks the ESPCI for hospitality while part of this work was completed. This work was partially supported by the Nanoscale Science and Engineering Initiative of the National Science Foundation under NSF Award Number CHE-0117752 and by the new York State Office of Science, Technology and Academic Reserach (NYSTAR).

\section{APPENDIX A: ZERO TEMPERATURE PHONON POLARIZATION}

At zero temperature the function $1-2 f(x)=\operatorname{sgn}(x)$, and the integral Eq. 109] and [10 may be performed explicitly. The corresponding expressions are

$$
\begin{gathered}
T_{1}\left(\mu_{L}, \omega\right)=\frac{1}{2 \pi\left(\omega^{2}+\Gamma^{2}\right)} \ln \frac{\left(\mu_{L}-\epsilon_{0}\right)^{2}+\frac{\Gamma^{2}}{4}}{\left(\mu_{L}+\omega-\epsilon_{0}\right)^{2}+\frac{\Gamma^{2}}{4}} \\
+\frac{1}{2 \pi \omega}\left(\frac{2 \Gamma}{\omega^{2}+\Gamma^{2}}\right) \arctan \frac{\mu_{L}+\omega-\epsilon_{0}}{\Gamma / 2} \\
-\frac{2}{\pi \Gamma \omega}\left(\frac{\Gamma^{2}}{\Gamma^{2}+\omega^{2}}\right) \arctan \frac{\mu_{L}-\epsilon_{0}}{\Gamma / 2}
\end{gathered}
$$

and

$$
\begin{gathered}
T_{2}\left(\mu_{L}, \omega\right)=-\frac{1}{\pi\left(\omega^{2}+\Gamma^{2}\right)}\left(\arctan \frac{\mu_{L}+\omega-\epsilon_{0}}{\Gamma / 2}+\arctan \frac{\mu_{L}-\epsilon_{0}}{\Gamma / 2}\right) \\
+\frac{1}{\pi \omega}\left(\frac{\Gamma / 2}{\omega^{2}+\Gamma^{2}}\right) \ln \frac{\left(\mu_{L}-\epsilon_{0}\right)^{2}+\frac{\Gamma^{2}}{4}}{\left(\mu_{L}+\omega-\epsilon_{0}\right)^{2}+\frac{\Gamma^{2}}{4}} \\
\text { APPENDIX B: HIGH-T LIMIT OF PHONON } \\
\text { RATE EQUATIONS }
\end{gathered}
$$

\section{APPENDIX B: HIGH-T LIMIT OF PHONON RATE EQUATIONS}

In this section we shall show how for weak electron phonon coupling and at high-T the quantum Boltzmann equation for phonons (Eq. [124] [125] [126) reduces to the classical rate equations derived in Section II. For $T \gg \Gamma$, we can replace the lorentzian broadenings in Eqn 125 and 126 by $\delta$ functions i.e. $\left(\frac{\Gamma / 2}{x^{2}+\Gamma^{2} / 4} \rightarrow \pi \delta(x)\right)$. Moreover for weak electron-phonon couplings we may identify the probability of single occupancy of the dot to be given by its value in the absence of electron phonon coupling, i.e. $P^{1}=\frac{\sum_{a} \Gamma_{a} f\left(\epsilon_{0}-\mu_{a}\right)}{\Gamma}$, so that

$$
\begin{gathered}
R_{n \rightarrow n+1}=(n+1) \lambda^{2} \frac{1}{1+\frac{\Gamma^{2}}{4 \omega_{0}^{2}}}\left\{P^{1} \sum_{b} \Gamma_{b}\left(1-f\left(\epsilon_{0}-\omega_{0}-\mu_{b}\right)\right)\right. \\
\left.+P^{0} \sum_{b} \Gamma_{b} f\left(\epsilon_{0}+\omega_{0}-\mu_{b}\right)\right\}
\end{gathered}
$$

and

$$
\begin{aligned}
R_{n \rightarrow n-1}= & n \lambda^{2} \frac{1}{1+\frac{\Gamma^{2}}{4 \omega_{0}^{2}}}\left\{P^{1} \sum_{b} \Gamma_{b}\left(1-f\left(\epsilon_{0}+\omega_{0}-\mu_{b}\right)\right)\right. \\
& \left.+P^{0} \sum_{b} \Gamma_{b} f\left(\epsilon_{0}-\omega_{0}-\mu_{b}\right)\right\}
\end{aligned}
$$

Now the classical rate equations in Eq. 37 rewritten for the case of a single resonant level with $U=0$ take the form,

$$
\begin{aligned}
& \dot{P}_{n}^{0}=-P_{n}^{0} \sum_{a, m} R_{n, m}^{a, 0,1}+\sum_{a, m} P_{m}^{1} R_{m, n}^{a, 1,0} \\
& \dot{P}_{n}^{1}=-P_{n}^{1} \sum_{a, m} R_{n, m}^{a, 1,0}+\sum_{a, m} P_{m}^{0} R_{m, n}^{a, 0,1}
\end{aligned}
$$

where $R_{n, m}^{a, 0,1}$ etc are defined in Eqns. 61] and 62 with $U=0$. In the weak electron-phonon coupling limit, only transitions that change the phonon number at most by 1 are allowed. In this limit the $\Gamma_{n, n+1}^{a} \rightarrow(n+1) \lambda^{2} \Gamma_{a}$. Moreover we may again factorize the joint probability distribution of having $0 / 1$ electrons and $m$ phonons as $P_{m}^{0,1}=P^{0,1} P_{m}^{p h}$, After making these approximations in Eq. B3 and B4 and adding the two equations we obtain

$$
\begin{aligned}
\dot{P}_{n}^{p h} & =-P_{n}^{p h}\left(R_{n \rightarrow n+1}+R_{n \rightarrow n-1}\right) \\
& +P_{n+1}^{p h} R_{n+1 \rightarrow n}+P_{n-1}^{p h} R_{n-1 \rightarrow n}
\end{aligned}
$$

with the rates given by Eq. B1 and B2
1 J. Chen, M. A. Reed, A. M. Rawlett, and J. M. Tour, Science, 286,1550 (1999); C.P. Collier, G. Mattersteig, E. W.
Wong, Y. Luo, K. Beverly, J, Sampaio, F. M. Raymo, J. F. Stoddart, and J. R. Heath, Science,289, 1172 (2000); 
W. Liang, M. P. Shores, M. Brockrath, J. R. Long and H. Park, Nature, 417, 725 (2002); J. Reichert, R. Ochs, D. Beckmann, H. B. Weber, M. Mayor, and H. v. Loheysen, Phys. Rev. Lett., 88, 176804 (2002); N. B. Zhitenev, H. Meng, and Z. Bao, Phys. Rev. Lett., 88, 226801, (2002); S. Kubatkin, A. Danilov, M. Hjort, J. Cornil, J. Bredas, N. Stuhr-Hansen, P. Hedegard and T. Bjornholm, Nature,425,68 (2003).

2 L. P. Kouwenhoven and C. M. Marcus, Phys. World 11 (6) , 35 (1998)and references therein. Also L. P. Kouwenhoven, C. M. Marcus, P.L. McEuen, S. Tarucha, R. M. Westervelt, and N. S. Wingreen Electron Transport in Quantum Dots Nato ASI conference proceedings, Series E345, ed. By L. P. Kouwenhoven, G. Schon, L.L. Sohn (Kluwer, Dordrecht, 1997).

3 see, e.g. Quantum effects in Coulomb blockade,I. L Aleiner, P. W. Brouwer and L. I Glazman, Physics Reports, 358, 309 (2002) and references therein.

4 J. Park, A. N. Pasupathy, J. I. Goldsmith, C. Chang, Y. Yaish, J. R. Petta, M. Rinkoski, J. P. Sethna, H Abruna, P. L. McEuen, and D. C. Ralph,Nature, 17, 722 (2002).

${ }^{5}$ H. Park, J. Park, A. K. L. Lim, E. H. Anderson, A. P. Alivisatos, and P. L. McEuen, Nature, 407, 57 (2000).

6 A. N. Pasupathy, J. Clark, C. Chang, A. V. Soldatov, S. Lebedkin, R. C. Bialczak, J. E. Grose, L. A. K. Donev, J. P. Sethna, D. C. Ralph and P. L. McEuen, cond-mat/0311150

7 L. Glazman and R. I. Shekhter, Zh. Eksp. Teor. Fiz. 94 292 (1987) [Sov. Phys. JETP 67163 (1988)].

8 N. S. Wingreen, K. W. Jacobsen, and J. W. Wilkins, Phys. Rev. B., 40, 11834 (1989).

9 U. Lundin and H. McKenzie, Phys. Rev. B., 66, 075303 (2002).

10 J. X. Zhu and A. V. Balatsky, Phys. Rev. B 67, 165326
(2003).

11 K. D. McCarthy, N. Prokofev, and M. T. Tuominen, Phys. Rev. B, 67, 245415 (2003).

12 V. Aji, J. E. Moore, and C. M. Varma, cond-mat/0302222

13 A. O. Gogolin and A. Komnik, unpublished (cond-mat/0207513).

14 A. S. Alexandrov and A. M. Bratkovsky, and R. S. Williams, Phys. Rev. B. 67, 075301 (2003); S. Alexandrov and A. M. Bratkovsky, Phys. Rev. B. 67, 235312 (2003).

15 Karsten Flensberg, Phys. Rev. B, 68, 205323 (2003).

16 D. Fedorets, cond-mat/0311104 D. Fedorets, L. Y. Gorelik, R. I. Shekhter, and M. Jonson, cond-mat/0311105 D. Fedorets, L. Y. Gorelik, R. I. Shekhter, and M. Jonson, Europhys. Let. 58, 99 (2002).

17 C. W. Gardiner and P. Zoller, Quantum Noise, Springer Verlag, 2nd Edition.

18 A. Jauho, N. S. Wingreen, and Y. Meir, Phys. Rev. B. 50, 5528 (1994).

19 See, for example L. I. Glazman and K. Matveev, Pis'ma Zh. Eksp. Theor. Fiz. 48403 (1988) or C. W. J. Beenakker, Phys. Rev. B, 44,1646 (1991).

20 See, e.g. section 4.3 of G. D. Mahan, Many Particle Physics, 2nd Edition, Plenum Publishers.

21 A. N. Korotkov, Phys. Rev. B 49, 10381 (1994).

22 L. V. Keldysh, Zh. Eksp. Teor. Fiz., 47, 1945 (1964) [Sov. Phys. JETP 46, 155 (1977)].

23 M. G. Vavilov and I. L. Aleiner, Phys. Rev. B, 69, 035303 (2004).

24 H. Schoeller, and G. Schøn, Phys. Rev. B, 50, 18436 (1994).

25 J. Rammer, Rev. Mod. Phys. 63, 781 (1991).

26 S. Braig and K. Flensberg, cond-mat/0401347 